\title{
Catalogue of Danish Alysiinae (Hymenoptera: Braconidae), with the description of two new species of Aspilota Foerster, 1863
}

\author{
Francisco Javier PERIS-FELIPO ${ }^{1, *}$, Sergey A. BELOKOBYLSKIJ ${ }^{2}$, \\ Lars VILHELMSEN ${ }^{3} \&$ Thorkild MUNK ${ }^{4}$ \\ ${ }^{1}$ Bleichestrasse 15, CH-4058 Basel, Switzerland. \\ ${ }^{2}$ Zoological Institute of the Russian Academy of Sciences, St Petersburg, 199034, Russia; Museum \\ and Institute of Zoology of the Polish Academy of Sciences, Wilcza 64, Warszawa 00-679, Poland. \\ ${ }^{3}$ Zoological Museum, Natural History Museum of Denmark, SCIENCE, University of Copenhagen. \\ Universitetsparken 15, Copenhagen Ø, DK-2100 Denmark. \\ ${ }^{4}$ Deceased 24 Dec. 2013, former address: Natural History Museum Aarhus, Denmark. \\ *Corresponding author: peris.felipo@gmail.com \\ 2 Email: doryctes@gmail.com \\ ${ }^{3}$ Email: 1bvilhelmsen@snm.ku.dk \\ * urn:Isid:zoobank.org:author:C7B698F7-0A6A-4C4A-915F-8D5ACC380853 \\ ${ }^{2}$ urn:Isid:zoobank.org:author:13EDEDEF-68BA-430B-8FC3-0096874859AB \\ ${ }^{3}$ urn:Isid:zoobank.org:author:96FC3783-9FA7-421E-B292-6718A3762D45 \\ ${ }^{4}$ urn:1sid:zoobank.org:author:01C54836-6431-4292-8A9E-C75679E85C7C
}

\begin{abstract}
In the present study, a total of 153 species of Alysiinae (Hymenoptera: Braconidae) from Denmark are catalogued. Two species are described as new for science: Aspilota leptoarticulata Munk \& Peris-Felipo sp. nov. and A. grandis Munk \& Peris-Felipo sp. nov. Additionally, 38 alysiine species are recorded for the first time for the Danish fauna. A faunistic list with distribution data and host records is provided.
\end{abstract}

Keywords. Parasitoids, Alysiinae, faunistic list, new species, Denmark.

Peris-Felipo F.J., Belokobylskij S.A., Vilhelmsen L. \& Munk T. 2016. Catalogue of Danish Alysiinae (Hymenoptera: Braconidae), with the description of two new species of Aspilota Foerster, 1863. European Journal of Taxonomy 250: 1-48. http://dx.doi.org/10.5852/ejt.2016.250

\section{Introduction}

The Alysiinae is a subfamily of Braconidae, comprising more than 100 genera and 2300 species worldwide (Yu et al. 2012). They are koinobiont endoparasitoids of Diptera-Cyclorrapha (Gimeno et al. 1997; Zaldivar-Riverón et al. 2006; Peris-Felipo et al. 2014a); records of some species of Hymenoptera and Lepidoptera as hosts (Yu et al. 2012) are most probably erroneous. Some alysiine species can be relevant from an economic point of view due to their potential role in pest control (González et al. 2000; Elpino-Campos et al. 2007). The monophyly of the Alysiinae is based on the following distinctive 
apomorphic characters: the shape, increased number of teeth and position of the exodont mandibles and complete loss of the occipital and prepectal carinae. The subfamily is divided into two large and polymorphic tribes, the Alysiini and Dacnusini (Shenefelt 1974; Yu et al. 2012). Morphologically, these two tribes are mainly separated by the presence (Alysiini) or absence (Dacnusini) of the fore wing vein cuqu2 (r-m or second radiomedial); accordingly, Alysiini has two submarginal (radiomedial) cells while Dacnusini has only one cell (Shenefelt 1974; Yu et al. 2012; Peris-Felipo et al. 2014a). Members of the tribe Alysiini are common parasitoids of Diptera-Cyclorrapha, usually inhabiting humid and ephemeral substrates. Dacnusini are almost exclusively specialized on leaf and stem miners, mainly of the families Agromyzidae, Ephydridae and Chloropidae (Belokobylskij 2005; Berry 2007; Fischer \& Beyarslan 2012; Peris-Felipo et al. 2014a). Species of the Alysiini genera Aphaereta Foerster, 1863 and Alysia Latreille, 1804 have been used in biological control programs, among others of Calliphoridae and Muscidae, while some Dacnusini have been successfully employed to control pests of leaf-mining flies and Tephritidae (Wharton et al. 1997).

Several investigations of the Alysiinae fauna have been conducted for the territories of northern Europe: among them, Tobias (1962) - North-West of Russia; Königsmann (1969) - Denmark, Finland, Norway and Sweden; Hedqvist (1972, 1973) - Sweden; Gannota (1993), Koponen (1999, 2000), Koponen \& Vikberg (2013) and van Achterberg \& Vikberg (2014) - Finland; van Achterberg \& Koponen (2003) Finland, Estonia; Riedel et al. (2002) - Norway; Munk et al. (2013a, 2013b) - Denmark, Finland; PerisFelipo et al. (2014a, 2014b) - Denmark, Finland, Norway, Sweden. No survey focused on the Danish fauna has been published until now, but the publications on the faunas of neighbouring countries serve as a starting point for an estimation of the Danish alysiine diversity. Adjacent countries, such as Germany (337 alysiine species), Poland (231) and Sweden (234) are better known (Yu et al. 2012). However, until now only 114 species of 20 genera were known in Denmark (Yu et al. 2012; van Achterberg 2013; Peris-Felipo et al. 2014a, 2014b). Thorkild Munk collected and identified specimens of Alysiinae during many years throughout the country, but the majority of this material remained unpublished at the time

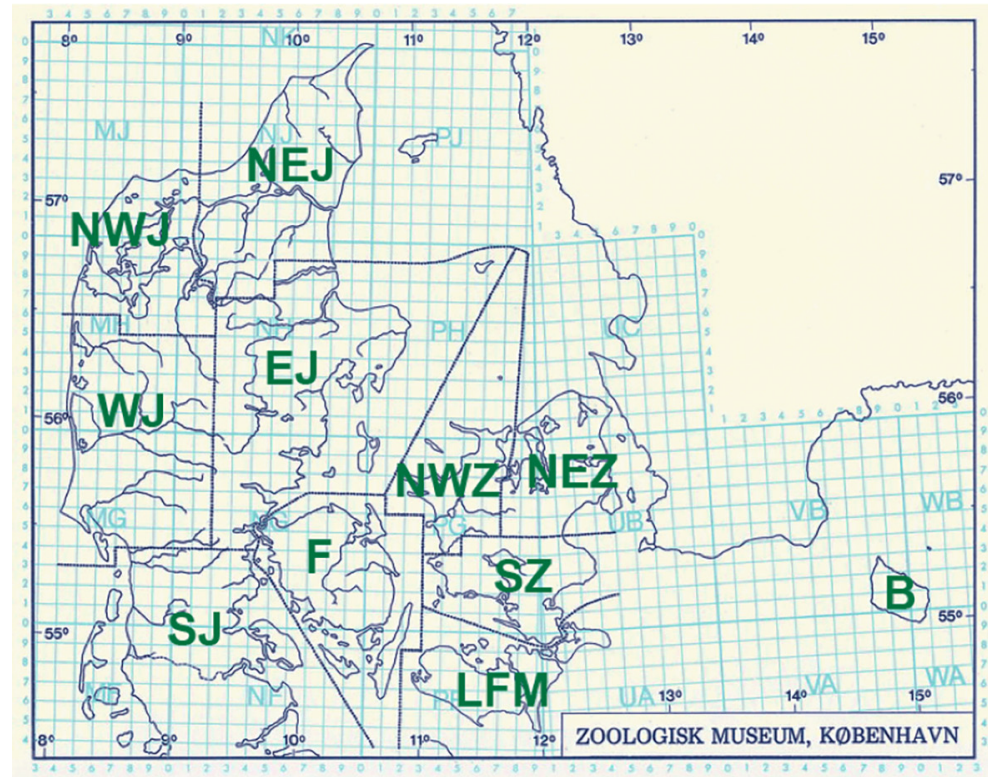

Fig. 1. Map of Denmark (excluding Greenland and the Faroe Islands) showing the eleven faunistic districts. $\mathrm{B}=$ Bornholm; EJ = East Jutland (Jylland); F = Funen (Fyn); LFM = Lolland - Falster - Møn; NEJ = North East Jutland; NEZ = North East Zealand (Sjælland); NWJ = North West Jutland; NWZ = North West Zealand; SJ = South Jutland; SZ = South Zealand; WJ = West Jutland. 
his death in December 2013. The current catalogue of Danish Alysiinae is mainly based on the results of the study of his extensive material.

\section{Material and methods}

\section{Area of study}

Denmark is located in northern Europe between the Baltic and the North Seas, comprising the southwestern part of Scandinavia. It is the smallest country of this region, having a total area of $43094 \mathrm{~km}^{2}$. In contrast, Denmark has a comparatively long coastline, and no point of the country is more than $52 \mathrm{~km}$ from the sea. The main part (almost $3 / 4$ of the territory) is the Jutland Peninsula (Fig. 1: NEJ, NWJ, EJ, WJ, SJ) attached to northern Germany. The remainder of the country is an archipelago comprising more than 1400 islands above $100 \mathrm{~m}^{2}$; of these, 443 are named and 72 inhabited (Geodatastyrelsen 2016). Denmark is generally flat with only low elevation, having an average height above sea level of $31 \mathrm{~m}$; the highest natural point is Møllehøj at $170.86 \mathrm{~m}$ (Geodatastyrelsen 2016).

The climate is temperate and maritime, with mild winters (average temperature of $1.5^{\circ} \mathrm{C}$ ) and cool summers (average temperature $17.2^{\circ} \mathrm{C}$ ). Precipitation is fairly evenly distributed throughout the year, averaging $765 \mathrm{~mm}$ annually (Danish Metereological Institute 2016). Denmark is the most densely populated country in Scandinavia, and it is one of the most intensively cultivated countries in the world, about $66 \%$ of the area being used for agricultural purposes (Den Store Danske 2016). Forests comprise about $16 \%$ of the area, commercially run evergreen tree plantations making up a substantial part. Habitats that are not at least moderately affected by human activities are virtually non-existent.

\section{Sampling and identification}

Sampling was carried out by hand net and Malaise traps. Specimens were preserved in $75 \%$ alcohol until preparation. The criteria indicated by Tobias et al. (1986a, b), van Achterberg (1993), Sharkey \& Wharton (1997), Belokobylskij \& Tobias (2007) and Peris-Felipo et al. (2014a) were used for species identification. Classification follows Yu et al. (2012) and Peris-Felipo et al. (2014a). For the terminology of the morphological features, sculpture and measurements see Peris-Felipo et al. (2014a) and for wing venation nomenclature see Peris-Felipo et al. (2014a) and van Achterberg (1993). New records of species for the country are marked by an asterix $(*)$.

Most examined material and types of described species are deposited in the collection of the Natural History Museum of Denmark (Copenhagen, Denmark; NHMD), and some paratypes and additional material are housed in the Entomological Collection at the University of Valencia (Valencia, Spain; ENV), Naturhistorisk Museum Aarhus (Aarhus, Denmark; NMA), Zoological (Natural History) Museum, University of Helsinki (Helsinki, Finland; MZA); Naturhistorisches Museum Wien (Vienna, Austria; NHMW), Naturalis Biodiversity Center (Leiden, Netherlands; RMNH), Zoological Institute of the Russian Academy of Sciences (St Petersburg, Russia; ZISP) and Zoologische Staatssammlung München (Munich, Germany; ZSSM).

\section{Results}

A total of 153 species belonging to 23 genera (13 from Alysiini and 10 from Dacnusini), are recorded and catalogued for the Danish fauna. 
Subfamily Alysiinae Leach, 1815

Tribe Alysiini Leach, 1815

Genus Adelphenaldis Fischer, 2003

Material examined

*Adelphenaldis globipes (Fischer, 1962)

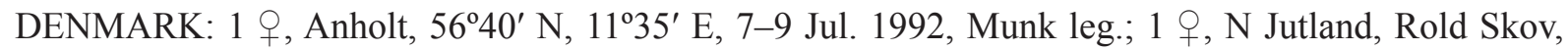

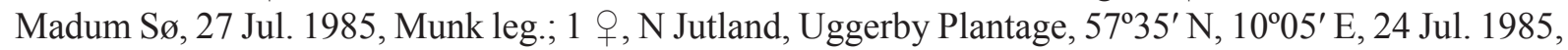
Munk leg.; 1 ㅇ, E Jutland, Nørreris Skov, 10 km NW of Aarhus, 16 Jul. 1986, Munk leg.

\section{Distribution}

Palaearctic Region (Yu et al. 2012). Denmark (first record).

Genus Alloea Haliday, 1833

Published material

Alloea contracta Haliday, 1833

Data obtained from Fauna Europea (van Achterberg 2013).

\section{Distribution}

Palaearctic Region (Yu et al. 2012).

Genus Alysia Latreille, 1804

Published material

Alysia (Alysia) incongrua Nees, 1834

“Copenhagen” (Wharton 1986).

\section{Distribution}

Western Palaearctic (Yu et al. 2012).

\section{Published material}

Alysia (Anarcha) subaperta Thomson, 1895

"Denmark" (Wharton 1988).

\section{Distribution}

Nearctic and Western Palaearctic (Yu et al. 2012).

\section{Material examined}

*Alysia (Anarcha) sophia Haliday, 1838

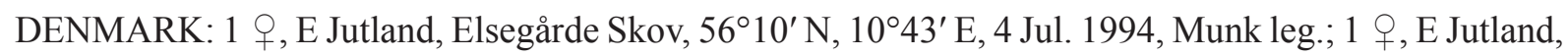
Ibæk, 5 km SE of Vejle, 15 Jun. 1989, Munk leg.; 7 q , E Jutland, Højen Bæk, 5 km S of Vejle, 30 Jun. 1982, 3, 5-7, 23 Jul. 1984 and 16 Jun. 1986, Munk leg.; 4 우, E Jutland, Klattrup, 5-7 km S of Vejle, 12 Jun. and 12-15 Jul. 1983, Munk leg.; 2 q 9 , E Jutland, Yding Skov, 56 $00^{\prime}$ N, 9 $9^{\circ} 48^{\prime}$ E, 28 Jun. 1985 and 27 Jun. 1987, Munk leg.; 3 우우, N Jutland, Krogens Mølle, 15 km SW of Frederikshavn, 23 Jun. 1982 and 11-13 Aug. 1986, Munk leg. 


\title{
Distribution
}

Palaearctic Region (Yu et al. 2012). Denmark (first record).

\author{
Genus Aspilota Forster, 1863
}

\section{Material examined}

*Aspilota delicata Fischer, 1973

DENMARK: 1 , , E Jutland, Grejsdal, N of Vejle, 9 Jul. 1987, Munk leg.; 1 q, E Jutland, Hørret Skov,

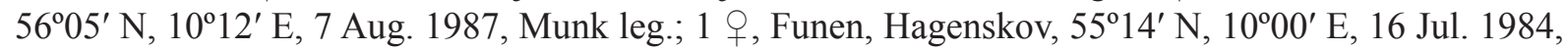
Munk leg.; 3 우, NE Zealand, Lystrup Skov, 1 Sep. 1984, Munk leg.; 1 ㅇ, E Jutland, Tirsbæk, 18 Aug. 1984, Munk leg.

\section{Distribution}

Palaearctic Region (Yu et al. 2012). Denmark (first record).

\section{Material examined}

*Aspilota furtnerana Fischer, 1973

DENMARK: 1 , , E Jutland, Ibæk, 5 km SE of Vejle, 15 Jun. 1989, Munk leg.; 2 + 9 , E Jutland, Højen Bæk, $5 \mathrm{~km} \mathrm{~S}$ of Vejle, 20 Jun. 1978, Munk leg.; 1 क, E Jutland, Klattrup, 7 km S of Vejle, 12 Jun. 1983, Munk leg.; 2 우, E Jutland, Staksrode Skov, 5541' N, 951' E, 11 Jun. 1984 and 17 Jul. 1987, Munk leg.

\section{Distribution}

Western Palaearctic (Yu et al. 2012). Denmark (first record).

\section{Material examined}

*Aspilota fuscicornis (Haliday, 1838)

DENMARK: $2 \hat{\jmath}$, Funen, Smedsholm, $3 \mathrm{~km}$ W of Fjelsted, 4 Jun. 1982, swept on Mercurialis community, Munk leg.; 1 ㅇ, E Jutland, 12 km SW of Sillerup, 25 Aug. 1986, Munk leg.; 4 우, 6 ふふ઼, E Jutland, Klattrup, $10 \mathrm{~km} \mathrm{~S}$ of Vejle, 4 Jun. 1978, 6 Jun., 12-15 Jul. and 29 Aug. 1982, swept on compost from the garden, 1 Aug. and 2 Sep. 1983, 24 Jul. 1984, Munk leg.; 1 9, E Jutland, NG 36, Viuf Skov, 21 Jun. 1986, Munk leg.; 1 क, E Jutland, NG 57, Rosenvold, N of Vejle Fjord, 9 Jun. 1985, Munk leg.; 2 우, 2 ふ઼઼, E Jutland, Sr Vissing, $17 \mathrm{~km} \mathrm{~W}$ of Skanderborg, 30 Jun. 1983, swept on old oak wood,

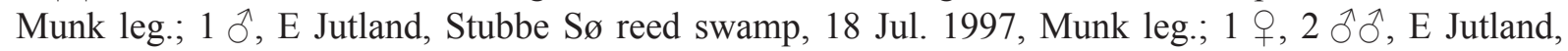
Tirsbæk, 30 Jul. 1983 and 18 Aug. 1984, Munk leg.; 1 , E Jutland, Yding Skov, SW of Skanderborg, 6 Sep. 1986, Munk leg.; 1 ㅇ, Mid-Jutland, Egtved Skov, 9 Aug. 1975, Munk leg.; 1 đ, N Jutland, Måstrup

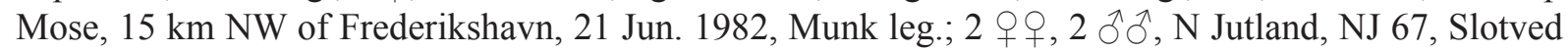
Skov, 20 Jun. 1982, 20 Jun. 1983, 18 Jun. and 7 Aug. 1984; 1 ㅇ, S Jutland, Arild, 1 Jul. 1986, Munk

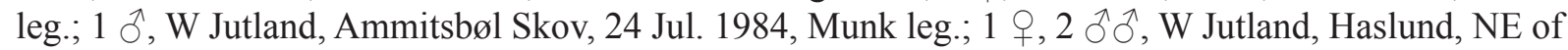
Ribe, 1-2 Jul. 1984, Munk leg.; 2 đ̊ ổ, E Jutland, Kjellerup, 56¹8’ N, $9^{\circ} 23$ E, 18 Aug. 1989, Munk leg.; 1 , , N Jutland, Lindknud, oak shrub, 13 Jul. 1987, Munk leg.; 1 ㅇ, 1 Oૈ, E Jutland, Ammitsbøl Skov, 55³8' N, 9²6' E, 29 Jul. 1984 and 15 Jun. 1986, Munk leg.; 1 ․, E Jutland, Børkop Skov, 12 Aug. 1982, swept on Mercurialis community, Munk leg.; 1 ․, E Jutland, Dråby Skov, 11 Jun. 1997, Munk leg.; 1 , E Jutland, Fuglslev Plantage, 56 $6^{\circ} 16^{\prime}$ N, 10 $0^{\circ} 43^{\prime}$ E, 10 Sep. 1988, Munk leg.; 1 \&, E Jutland, Grejsdal, Lerbæk Mølle, 14 Jun. 1986, Munk leg.; 1 + , E Jutland, Grejsdal, N of Vejle, 24 Aug. 1987, Munk leg.;

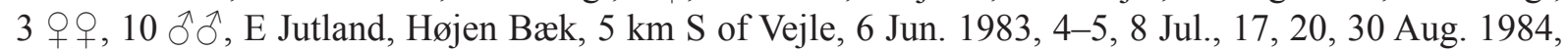
3 Jul. and 22 Aug. 1987, Munk leg.; 1 Ĵ, E Jutland, Mejlgård Skov, 17 May 2000, Munk leg.; 1 q, E Jutland, Nørreskov, 10 km E of Kolding, 18 Jul. 1982, Munk leg.; 1 + , E Jutland, Runkenbjerg, Vejle,

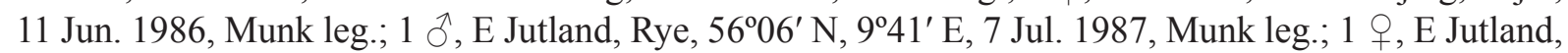




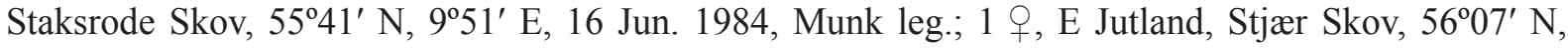

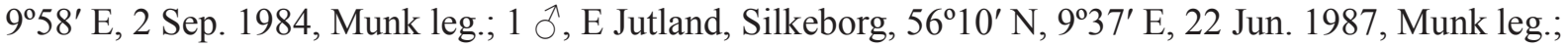

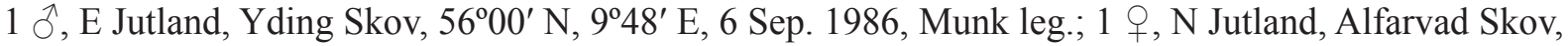

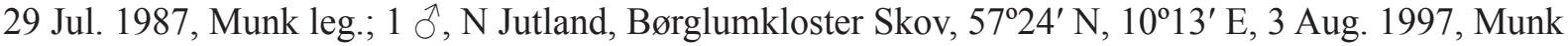
leg.; 1 § , N Jutland, near Rold Skov, 25 km S of Aalborg, 29 May 1998, Munk leg.; 1 ㅇ, N Jutland, Sindal, 57 $28^{\prime}$ N, $10^{\circ} 12^{\prime}$ E, 13 Aug. 1986, swept on compost, Munk leg.; 1 , , NW Jutland, Barnsø, Nors

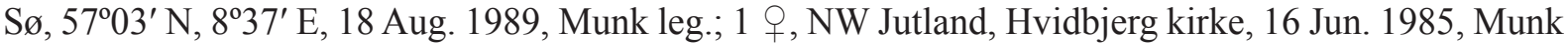

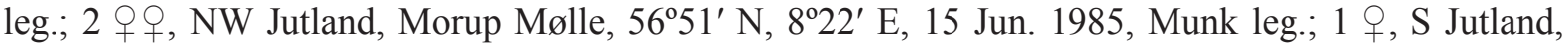

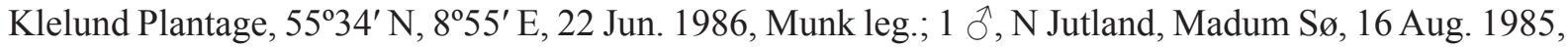

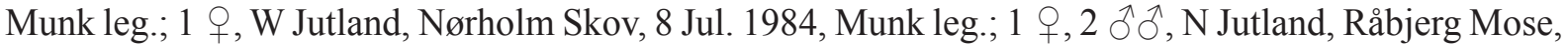
21 Jun. 1984, Munk leg.; 1 ô, N Jutland, Rold Skov, Mossø, 14 Aug. 1984, Munk leg.; 1 ô, N Jutland, Slotved Skov, 13-14 Jun. 1982, Munk leg.; 1 ठે, E Jutland, Tirsbæk, 18 Aug. 1984, Munk leg.; 1 q,

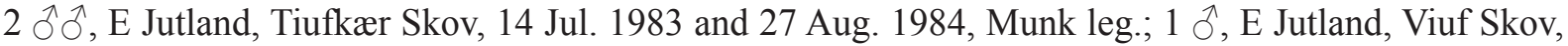
21 Jun. 1986, Munk leg.; 1 , E Jutland, Yding Skov, 19 Sep. 1985, Munk leg.

\section{Distribution}

Palaearctic region (Yu et al. 2012). Denmark (first record).

\section{Remarks}

Specimens of this species were sampled from different habitats such as Mercurialis community, oak wood or compost from gardens.

$$
\begin{aligned}
& \text { Aspilota grandis Munk \& Peris-Felipo sp. nov. } \\
& \text { urn:Isid:zoobank.org:act:DA6C33A7-925A-4C5D-97C2-36EBD92D12F2 }
\end{aligned}
$$

Figs 2-3

\section{Comparative diagnosis}

The new species is similar to A. imparidens Fischer, 1974 (Fischer 1974b) and A. laevinota Tobias, 1962 (Tobias 1962). Aspilota grandis sp. nov. differs from A. imparidens in having the first flagellar segment 3.3 times as long as its maximum width (7.0 times in $A$. imparidens), middle segments $1.3-$ 1.5 times as long as their maximum width (3.0 times in A. imparidens), hind femur 4.0 times as long as its maximum width (4.3 times in $A$. imparidens), and precoxal suture not reaching anterior or posterior margins of mesopleuron (reaching anterior margin in A. imparidens). Also, the new species differs from A. laevinota in having the face 1.8 times as wide as high (1.5 times in $A$. laevinota), the first flagellar segment 3.3 times as long as its maximum width (7.0 times in A. laevinota), middle segments 1.3-1.5 times as long as their maximum width (3.0 times in A. laevinota), and vein r2 (3-SR) 1.7 times as long as vein cuqu1 (2-SR) (1.5 times A. laevinota).

\section{Etymology}

The name is derived from Latin "grandis", meaning "big", referring to the size of the species.

\section{Type material}

Holotype

DENMARK: + , E Jutland, Højen Bæk, 5 km S of Vejle, 14 Jul. 1987, Munk leg. (NHMD).

Paratype

GERMANY: 1 §, Lippoldshausen, 20 May 1967, Haeselbarth leg. (ZSSM). 


\section{Description}

Female (holotype)

HEAD. In dorsal view twice as wide as its median length, 1.4 times as wide as mesoscutum, with rounded temples behind eyes. Maximum width of head at level of temple (dorsal view) larger than width at level of eyes. Eye in lateral view 1.6 times as high as wide and 0.9 times as wide as temple medially. POL 1.3 times OD; OOL 3.6 times OD. Face 1.8 times as wide as high; inner margins of eyes subparallel.
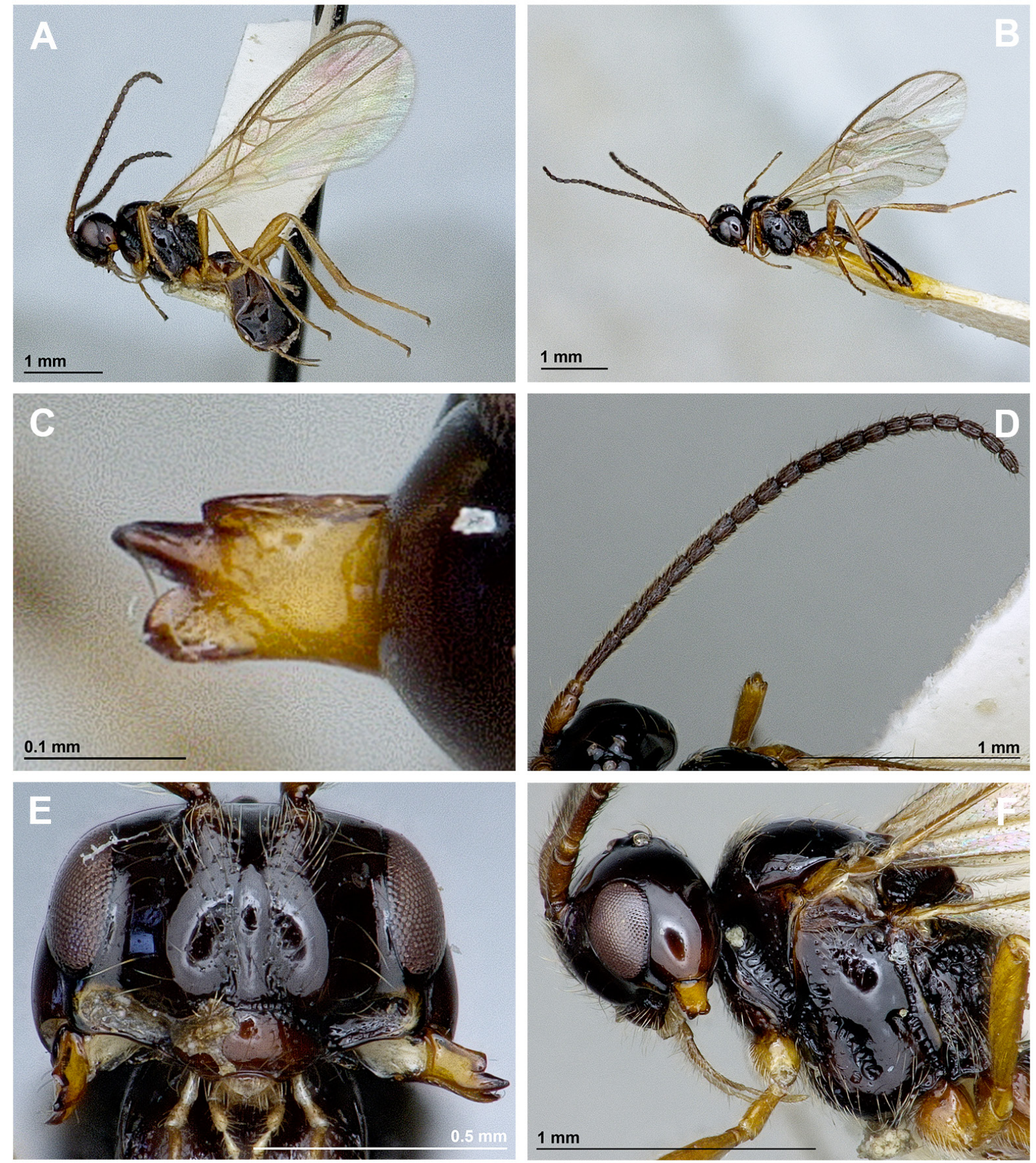

Fig. 2. Aspilota grandis Munk \& Peris-Felipo sp. nov. (A, C-F ค; B §). A-B. Habitus, lateral view. C. Mandible. D. Antenna. E. Head, frontal view. F. Head and mesosoma, lateral view. 
Clypeus slightly curved ventrally, 2.4 times as wide as high. Mandible widened towards apex, 1.7 times as long as maximum width. Upper tooth of mandible distinctly shorter than middle and lower teeth; middle tooth long and pointed; lower tooth longer than upper tooth, rounded apically. Antenna thick, 21 -segmented. Scape 3.0 times as long as pedicel. First flagellar segment 3.3 times as long as its apical width, 1.6 times as long as second segment; $2^{\text {nd }}$ segment 2.0 times as long as its maximum width, $3^{\text {rd }}$ segment 1.8 times, $4^{\text {th }}$ to $8^{\text {th }} 1.5-1.6$ times, $9^{\text {th }}$ to $18^{\text {th }} 1.3-1.4$ times, and $19^{\text {th }}$ (apical) 1.8 times as long as their maximum width, respectively.

Mesosoma. In lateral view 1.2 times as long as high. Mesoscutum 1.1 times as long as maximum width. Notauli mainly absent on horizontal surface of mesoscutum. Mesoscutal pit absent. Prescutellar depression smooth, with median and lateral carinae. Precoxal suture present, reaching anterior margin of mesopleuron. Posterior mesopleural furrow crenulate. Propodeum sculptured, without areola. Propodeal spiracle small.
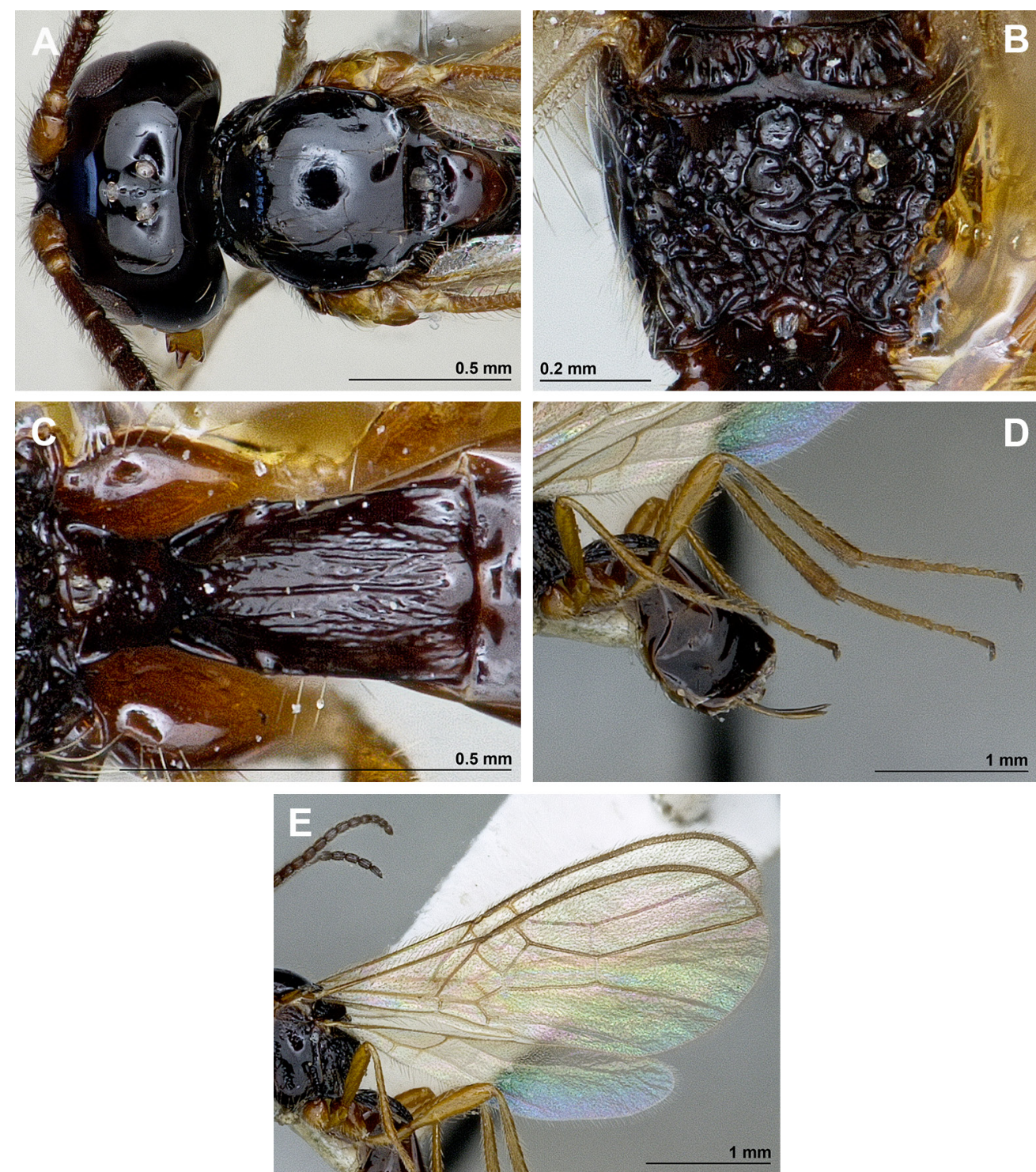

Fig. 3. Aspilota grandis Munk \& Peris-Felipo sp. nov. ()). A. Head and mesonotum, dorsal view. B. Propodeum, dorsal view. C. First metasomal tergite, dorsal view. D. Hind leg, metasoma and ovipositor. E. Fore and hind wings. 
Legs. Hind femur 4.0 times as long as its maximum width. Hind tibia slightly widened towards apex, 9.2 times as long as its maximum subapical width, about as long as hind tarsus. First segment of hind tarsus twice as long as second segment.

WiNGS. Length of fore wing 2.5 times its maximum width. Radial (marginal) cell ending at apex of wing, 3.5 times as long as its maximum width. Vein cuqu1 (2-SR) present and sclerotized. Vein r2 (3SR) 1.7 times as long as vein cuqu1 (2-SR); vein r3 (SR1) 2.5 times as long as vein r2 (3-SR). Nervulus (cu-a) distinctly postfurcal. Brachial (subdiscal) cell closed distally, 3.0 times as long as its maximum width. Hind wing 4.8 times as long as its maximum width.

Metasoma. Distinctly compressed. First tergite striate, slightly widened towards apex, twice as long as its apical width. Ovipositor as long as first tergite, distinctly shorter than metasoma, 0.8 times as long as hind femur.

Colour. Body, antenna and pterostigma dark brown. Mandible and legs brown. Wings hyaline.

LeNGTH. Body $3.1 \mathrm{~mm}$; fore wing $3.6 \mathrm{~mm}$; hind wing $2.8 \mathrm{~mm}$.

Male (paratype)

Body length $3.2 \mathrm{~mm}$. Fore wing length $3.6 \mathrm{~mm}$; hind wing length $2.7 \mathrm{~mm}$. Antenna 22-segmented. First flagellar segment 3.3 times as long as its maximum width. Hind femur 4.2 times as long as its maximum width. Otherwise similar to female.

\section{Distribution}

Denmark, Germany.

\section{Material examined}

*Aspilota hirticornis (Thomson, 1895)

DENMARK: 2 우, E Jutland, Fårup Sø, oak forest, 25 Jun. 1983, Munk leg.; 1 + , E Jutland, NG36, Børkop Skov, 1 Jul. 1982, Munk leg.; 1 9, S Jutland, Fovslet Skov, 55²6’ N, 9²4' E, 13 Jul. 1985, Munk leg.; 1 + , E Jutland, Klattrup Bygade, FB. HP., 24-25 Aug. 1982, Munk leg.; 1 क, E Jutland, Tiufkær Skov, 14 Jul. 1983, Munk leg.

\section{Distribution}

Western Palaearctic (Yu et al. 2012). Denmark (first record).

\section{Material examined}

*Aspilota imparidens Fischer, 1974

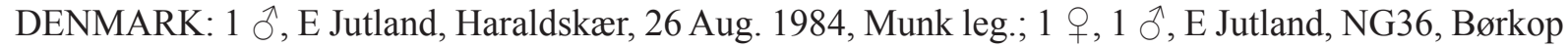
Skov, 1 Jul. and 12 Aug. 1982, Munk leg.; 1 , E Jutland, Højen Bæk, 5 km S of Vejle, 30 Jul. 1983, Munk leg.; 1 ð, E Jutland, Jelling, Fårup Sø, 28 Jun. 1983, swept on oak wood, Munk leg.; 2 우, 1 ○, E Jutland, Klattrup, $10 \mathrm{~km}$ S of Vejle, 11 Aug. 1982, swept on compost, 12 Jun. and 16 Sep. 1984, Munk

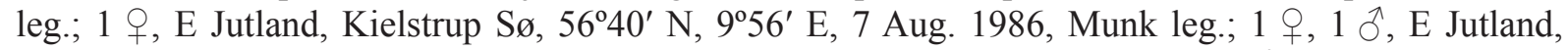
Tirsbæk, 5 km E of Vejle, 20 Jul. and 2 Aug. 1985, Munk leg.; 1 ð̊, N Jutland, NJ67, Åstrup Skov, 21 Jun. 1983, in oak plantation, Munk leg.; 1 đ̂, N Jutland, Måstrup Mose, NW of Frederikshavn, 21 Jun. 1982, Munk leg.; 1 đ, E Jutland, Klattrup, S of Vejle, 22 Jun. 1973, Munk leg.; 1 , E Jutland, Amaliegård

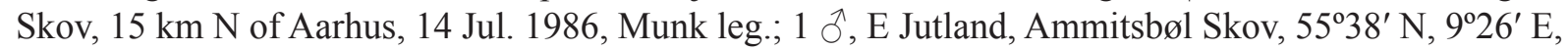
16 Jul. 1982, Munk leg.; 1 ㅇ, E Jutland, Grejsdal, Lerbæk, N of Vejle, 9 Aug. 1985, Munk leg.; 2 우, E Jutland, Højen Bæk, 5 km S of Vejle, 15 Jul. 1982 and 17 Aug. 1984, Munk leg.; 2 ồ , E Jutland, Højkol Skov, 56 05' N, 9³8' E, 7 Jul. 1986 and 21 Jul. 1988, Munk leg.; 1 ㅇ, E Jutland, Ibæk, 5 km 
SE of Vejle, 15 Jun. 1989, Munk leg.; 4 우, 1 ठ̊, E Jutland, Klattrup, 7 km S of Vejle, 10, 12 and 26 Jul., 1 Aug. 1982, Munk leg.; 1 क , E Jutland, Nørreris Skov, NW of Aarhus, 4 Sep. 1986, Munk leg.;

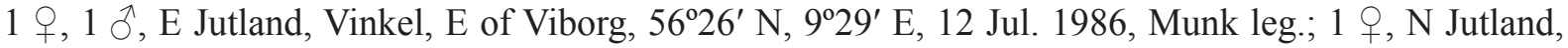

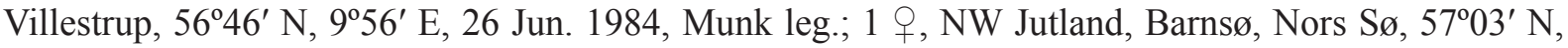
8037' E, 18 Aug. 1989, Munk leg.; 1 ㅇ, NW Jutland, Vilsbøl Plantage, 5702' N, 8³4' E, 20 Aug. 1989,

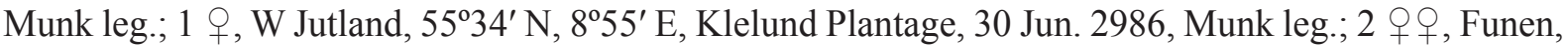
Smedsholm Skov, 3 km W of Fjelsted, 4 Jun. 1982, swept on Mercurialis community, Munk leg.; 1 , N Jutland, Vegger, 6 Aug. 1984, Munk leg.

\section{Distribution}

Western Palaearctic (Yu et al. 2012). Denmark (first record).

\section{Remarks}

Specimens were sampled from habitats such as oak wood and compost in gardens.

\section{Material examined}

*Aspilota insolita (Tobias, 1962)

DENMARK: 1 + , E Jutland, Højen Bæk, 5 km S of Vejle, 15 Jul. 1982, Munk leg.

\section{Distribution}

Western Palaearctic (Yu et al. 2012). Denmark (first record).

\section{Material examined}

*Aspilota intermediana Fischer, 1975

DENMARK: 1 ㅇ, W Jutland, Horsted, S of Vejle, 18 Oct. 1975, swept on champignon, Munk leg.; 1 , NE Zealand, Skibby, 21 May 1989 (P. Neerup Buhl leg.).

\section{Distribution}

Palaearctic Region (Yu et al. 2012). Denmark (first record).

\section{Remarks}

One specimen of this species was collected on Agaricus sp. (Fungi).

\section{Material examined}

*Aspilota iocosipecta Fischer, 1974

DENMARK: 1 + , E Jutland, Børskop Skov, SE of Vejle, 12 Jun. 1982, Munk leg.; 1 Oૈ, E Jutland,

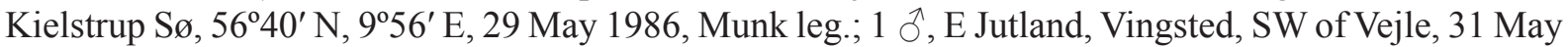

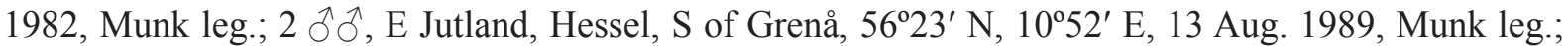
4 우, 10 $\widehat{\jmath}$, E Jutland, Højen Bæk, 5 km S of Vejle, 21 May, 1 and 27 Jun., 4 and 17 Jul., 30 Aug. 1983, 13 and 19 Jul., 23 and 30 Aug. 1984, 4 Jun. 1985, 9 and 21 Jun. 1986, Munk leg.; 1 q, E Jutland, Rugård Sønderskov, 56 $6^{\circ} 16^{\prime}$ N, 1049' E, 10 Jun. 1988, Munk leg.; 1 ㅇ, N Jutland, near Rold Skov, 25 km S of Aalborg, 14 May 2000, Munk leg.; 1 +, NW Jutland, Mønsted Skov, 17 Jun. 1984, Munk leg.

\section{Distribution}

Western Palaearctic (Yu et al. 2012). Denmark (first record). 


\section{Material examined}

*Aspilota iuxtanaeviam Fischer, 1980

DENMARK: 1 q, E Jutland, Klattrup, 7 km S of Vejle, 4 Aug. 1983, Munk leg.

\section{Distribution}

Western Palaearctic (Yu et al. 2012). Denmark (first record).

\section{Material examined}

*Aspilota laevinota Tobias, 1962

DENMARK: 1 ô, N Jutland, NJ67, Åstrup Skov, 19 Jun. 1984, Munk leg.

\section{Distribution}

Western Palaearctic (Yu et al. 2012). Denmark (first record).

Aspilota leptoarticulata Munk \& Peris-Felipo sp. nov. urn:1sid:zoobank.org:act:10CD8348-1EAF-41B8-B062-8BCB18B045DC

Figs 4-5

\section{Comparative diagnosis}

Apsilota leptoarticulata sp. nov. is similar to A. deserta Papp, 1967 (Fischer 1974a), A. makita Papp, 2008 (Papp 2008), and A. ventasa Belokobylskij, 2007. The new species differs from $A$. deserta in having the first flagellar segment 4.5 times as long as its maximum width (6.0 times in $A$. deserta), hind femur 4.5 times as long as its maximum width (3.6 times in $A$. deserta), precoxal suture present, not reaching anterior or posterior margins of mesopleuron (reaching anterior margin in $A$. deserta), and vein r2 (3-SR) 2.8 times as long as vein cuqu1 (2-SR) (1.5 times in $A$. deserta). On the other hand, A. leptoarticulata sp. nov. differs from A. makita (see Papp 2008) in having the first flagellar segment 4.5 times as long as its maximum width (4.0 times in A. makita), eye in lateral view 1.1 times as wide as temple medially ( 0.9 times in $A$. makita), hind femur 4.5 times as long as its maximum width (3.8 times in $A$. makita), and vein r2 (3-SR) 2.8 times as long as vein cuqu1 (2-SR) (1.7 times in A. makita). Finally, the new species differs from $A$. ventasa in having the first flagellar segment 4.5 times as long as its maximum width (3.7-3.8 times in $A$. ventasa), first metasomal tergite 2.0 times as long as its apical width (2.3 times in $A$. ventasa), eye in lateral view 1.1 times as wide as temple medially ( 0.9 times in $A$. ventasa), face 1.5 times as wide as high (1.8 times in $A$. ventasa), precoxal suture not reaching anterior margin of mesopleuron (reaching in $A$. ventasa), and vein r2 (3-SR) 2.8 times as long as vein cuqu1 (2-SR) (1.7 times in $A$. ventasa).

\section{Etymology}

The name refers to the long, thin flagellar segments.

\section{Type material}

\section{Holotype}

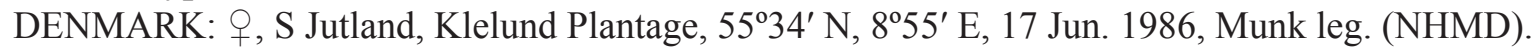

\section{Paratypes}

DENMARK: 1 đૈ, E Jutland, Dråby Strand, 5 Jun. 1997, Munk leg. (NHMD); 1 đ, E Jutland, 2 km E of Visborg, 28 Jul. 1997, Munk leg. (NHMD).

FINLAND: 1 †, ES Mikkelin mlk., 6859: 573, 23 Aug. 1975, Koponen leg. (MZA); 1 q, U. Nurmijärvi, 6715: 776, 4 Sep. 1976, Koponen leg. (MZA). 


\section{Description}

Female (holotype)

LeNGTH. Body $1.5 \mathrm{~mm}$; fore wing $2.0 \mathrm{~mm}$; hind wing $1.4 \mathrm{~mm}$.

HEAD. In dorsal view 1.8 times as wide as its median length, 1.4 times as wide as mesoscutum, with rounded temples behind eyes. Maximum width of head at level of temple (dorsal view) not larger than width at level of eyes. Eye in lateral view 1.6 times as high as wide and 1.1 times as wide as temple medially. POL 1.5 times OD; OOL 4.5 times OD. Face 1.5 times as wide as high; inner margins of eyes subparallel. Clypeus slightly curved ventrally, 2.5 times as wide as high. Mandible widened towards apex, 1.8 times as long as maximum width. Upper tooth of mandible distinctly shorter than middle and lower teeth; middle tooth long and pointed; lower tooth longer than upper tooth, rounded apically. Antenna slender, 16-segmented. Scape 1.5 times as long as pedicel. First flagellar segment 4.5 times as long as its apical width, 1.1 times as long as second segment; $2^{\text {nd }}$ and $3^{\text {rd }}$ segments 4.2 times as long as
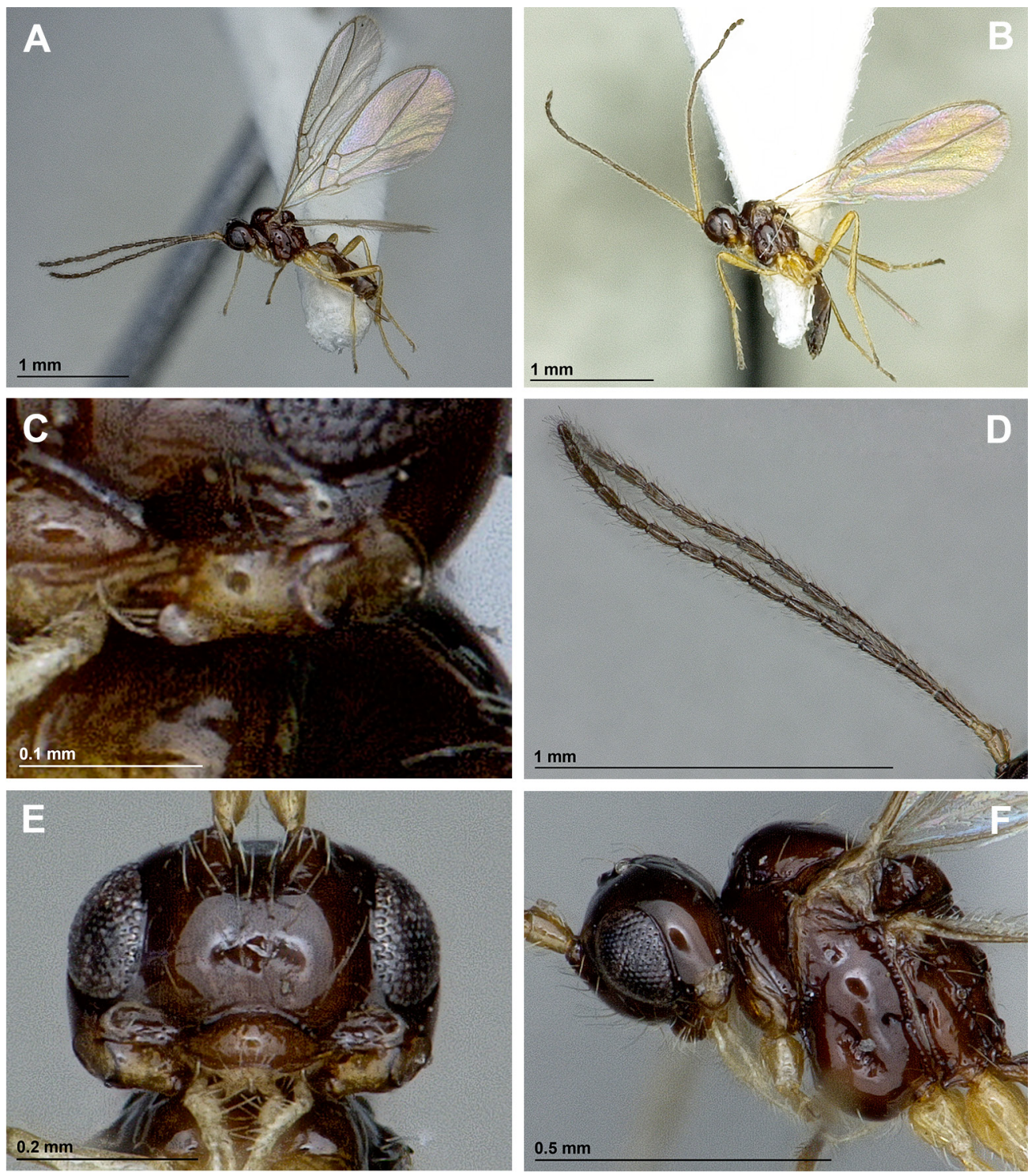

Fig. 4. Aspilota leptoarticulata Munk \& Peris-Felipo sp. nov. (A, C-F $q$; B đ). A-B. Habitus, lateral view. C. Mandible. D. Antennae. E. Head, frontal view. F. Head and mesosoma, lateral view. 
maximum width, $4^{\text {th }}$ and $5^{\text {th }}$ segments 3.8 times, $6^{\text {th }}$ to $9^{\text {th }}$ segments 3.3 times, $10^{\text {th }}$ segment 2.7 times, $11^{\text {th }}$ and $12^{\text {th }}$ segments 2.3 times, $13^{\text {th }}$ segment 2.0 times, and $14^{\text {th }}$ (apical) segment 2.2 times as long as their maximum widths.

Mesosoma. In lateral view 1.3 times as long as high. Mesoscutum as long as maximum width. Notauli mainly absent on horizontal surface of mesoscutum. Mesoscutal pit absent. Prescutellar depression smooth, without lateral carinae. Precoxal suture present, not reaching anterior or posterior margins of mesopleuron. Posterior mesopleural furrow crenulate. Propodeum sculptured, with large areola. Propodeal spiracle small.

Legs. Hind femur 4.5 times as long as its maximum width. Hind tibia slightly widened towards apex, about 8.0 times as long as its maximum subapical width, as long as hind tarsus. First segment of hind tarsus 1.9 times as long as second segment.
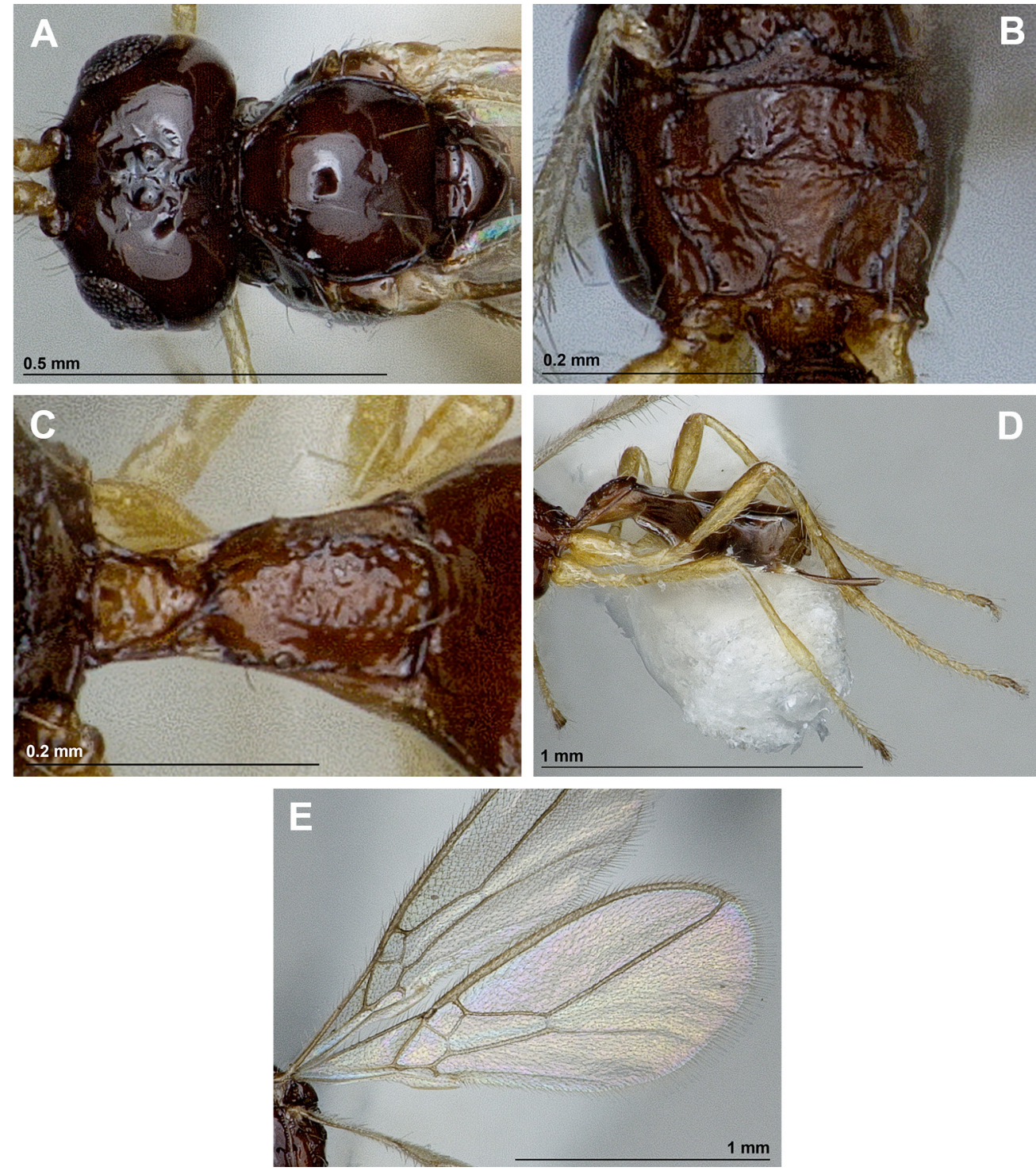

Fig. 5. Aspilota leptoarticulata Munk \& Peris-Felipo sp. nov. (†). A. Head and mesonotum, dorsal view. B. Propodeum, dorsal view. C. First metasomal tergite, dorsal view. D. Hind leg, metasoma and ovipositor. E. Fore wings. 
WINGS. Length of fore wing 2.4 times its maximum width. Radial (marginal) cell ending at apex of wing, 4.1 times as long as its maximum width. Vein cuqu1 (2-SR) present and sclerotized. Vein r2 (3SR) 2.8 times as long as vein cuqu1 (2-SR); vein r3 (SR1) 2.6 times as long as vein r2 (3-SR). Nervulus (cu-a) distinctly postfurcal. Brachial (subdiscal) cell closed distally, 2.8 times as long as its maximum width. Hind wing 6.0 times as long as its maximum width.

Metasoma. Distinctly compressed. First tergite finely rugose, slightly widened towards apex, twice as long as its apical width. Ovipositor 1.1 times as long as first tergite, distinctly shorter than metasoma, 0.8 times as long as hind femur.

Colour. Body, antenna and pterostigma brown to dark brown. Scape, pedicel, first flagellar segment, mandible and legs yellow. Wings hyaline.

VARIATION. Body length 1.4-1.6 mm.

\section{Male}

Body length $1.6 \mathrm{~mm}$. Fore wing length $2.0 \mathrm{~mm}$; hind wing length $1.5 \mathrm{~mm}$. Antenna 17-segmented. First flagellar segment 5.0 times as long as its maximum width; middle segments 3.0 times as long as their maximum widths. Otherwise similar to female.

\section{Distribution}

Denmark, Finland.

\section{Material examined}

*Aspilota nidicola Hedqvist, 1972

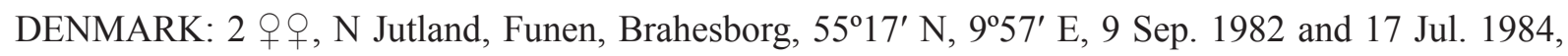
Munk leg.; 1 ㅇ, N Jutland, Frederikshavn Vandværksskoven, 2 Aug. 1987, Munk leg.

\section{Distribution}

Northern Palaearctic (Yu et al. 2012). Denmark (first record).

\section{Material examined}

*Aspilota pillerensis Fischer, 1973

DENMARK: 1 , E Jutland, Egå, 10 km NE of Aarhus, 13-19 Sep. 1984, Munk leg.; 1 , E Jutland, Højen Bæk, 5 km S of Vejle, 6 Jun. 1983, Munk leg.

\section{Distribution}

Western Palaearctic (Yu et al. 2012). Denmark (first record).

\section{Published material}

$$
\text { Aspilota ruficornis (Nees, 1834) }
$$

“Als af Oberlehrer Wüstnei” (Thomson 1895).

\section{Material examined}

DENMARK: 9 우, 4 $\widehat{\jmath}$, E Jutland, Egå, 10 km NE of Aarhus, 19-25 Sep. 1984, Munk leg.; 1 q, E Jutland, Højen Bæk, 5 km S of Vejle, 1 Aug. 1977, Munk leg.; 1 \%, E Jutland, Hjelm, 56 $08^{\prime}$ N,

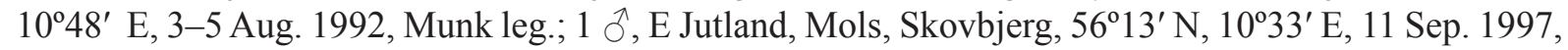
Munk leg. 


\section{Distribution}

Palaearctic region (Yu et al. 2012).

\section{Published material}

Aspilota spiricula Munk \& Peris-Felipo, 2014

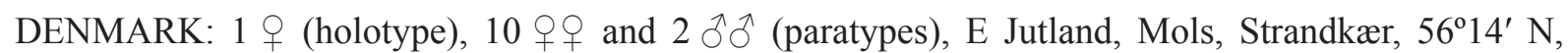
$10^{\circ} 25^{\prime}$ E, 30 Sep. 1982, Munk leg. (ENV, NMA, ZISP); 3 우우 (paratypes), same locality, but 29 Sep. 1986 and 2-3 Oct. 1991, Munk leg. (ENV, ZISP); 3 우우 and $1 \delta^{\widehat{ }}$ (paratypes), E Jutland, Højkol Skov,

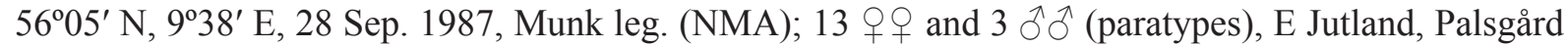

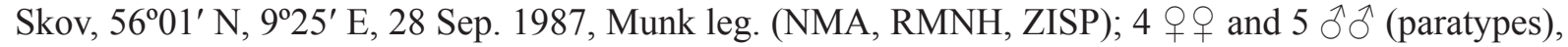

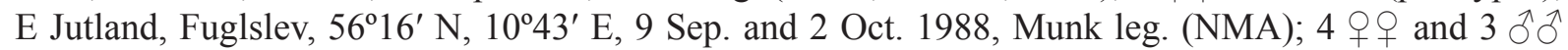

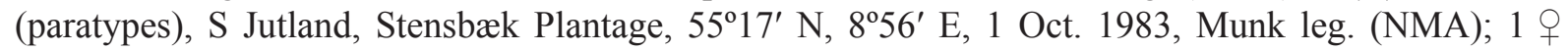
(paratype), S Jutland, Draved Skov, 1 Oct. 1983, Munk leg. (NMA); 3 o 9 and 2 ồ (paratypes), SW Jutland, Skallingen, near the field laboratory, 2 Oct. 1983, Munk leg. (NMA) (Peris-Felipo et al. 2014b).

\section{Distribution}

Western Palaearctic (Peris-Felipo et al. 2014b). Only known from Denmark.

\section{Material examined}

*Aspilota stenogaster Stelfox \& Graham, 1951

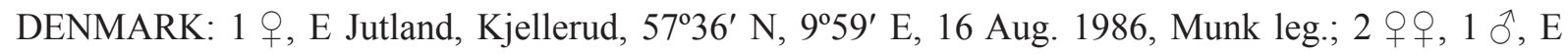
Jutland, Højkol Skov, 5605' N, 9³8' E, 21 Jul. 1988, Munk leg.; 1 đ̊, W Jutland, Højmose, 11 Oct. 1986, Munk leg.

\section{Distribution}

Palaearctic Region (Yu et al. 2012). Denmark (first record).

\section{Material examined}

*Aspilota styriaca Fischer, 1973

DENMARK: 1 q, E Jutland, Skramsø Plantage, Langsø W, 6 Jun. 2003, Munk leg.; 1 q, N Jutland, Bøgsted Plantage, $57^{\circ} 28^{\prime} \mathrm{N}, 10^{\circ} 07^{\prime}$ E, 25 Jun. 1982, Munk leg.; 4 우, N Jutland, Tværsted Plantage, $57^{\circ} 36^{\prime} \mathrm{N}, 10^{\circ} 43^{\prime} \mathrm{E}, 17$ and 20 Jun. 1982, Munk leg.

\section{Distribution}

Western Palaearctic (Yu et al. 2012). Denmark (first record).

\section{Material examined}

\section{*Aspilota tetragona Fischer, 1976}

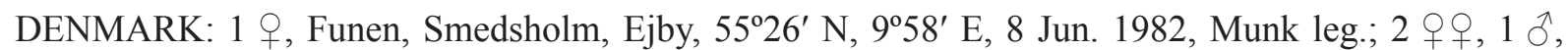
E Jutland, Klattrup, $10 \mathrm{~km} \mathrm{~S}$ of Vejle, 4 Aug. 1982 (19), swept on wet meadow, 12-13 Jul. 1983, swept on wood, Munk leg.; 1 ठิ, E Jutland, NH74, Mygind Skov, 19 Jul. 1989, Munk leg.; 1 ô, E Jutland, Palsgård, N of Juelsminde, 8 Jul. 1983, swept on wood, Munk leg.; 1 ðે, E Jutland, 17 km W

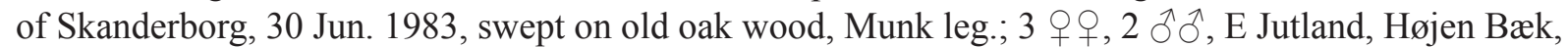
$5 \mathrm{~km} \mathrm{~S}$ of Vejle, 15 and 17 Jul. 1982, 7 Jul. and 25 Aug. 1984, 3 Jul. 1987, Munk leg.; 1 9, E Jutland,

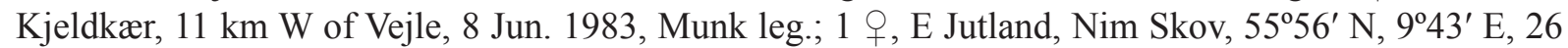

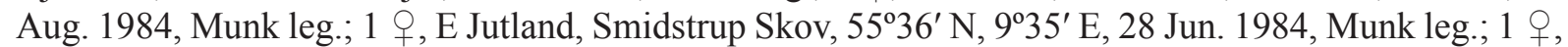
E Jutland, Vingsted, W of Vejle, 8 Jun. 1983, Munk leg.; 1 \%, N Jutland, Rævdal, 57 $23^{\prime}$ N, $10^{\circ} 24^{\prime}$ E, 16 Jun. 1984, Munk leg.; 1 क, NW Jutland, Morup Mølle, 5651' N, 8²2' E, 15 Jun. 1985, Munk leg. 


\section{Distribution}

Western Palaearctic (Yu et al. 2012). Denmark (first record).

\section{Remarks}

One specimen was sampled from wet meadow and two specimens from oak wood.

\section{Material examined}

*Aspilota ventasa Belokobylskij, 2007

DENMARK: 1 \%, Lolland, Errindlev, 25 May 1990, Munk leg. (NHMD); 1 ð̊, E Jutland, Ammitsbøl

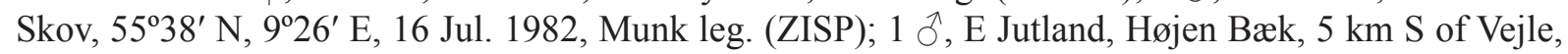
22 Jul. 1983, Munk leg. (NHMD); 1 ð̄, E Jutland, Nørreskov, 10 km E of Kolding, 18 Jul. 1982, Munk

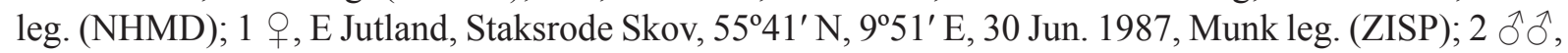
E Jutland, Tiufkær Skov, 22 Aug. 1978, Munk leg. (NHMD, ZISP); 1 ð̃, N Jutland, Terpet, S of Tårs,

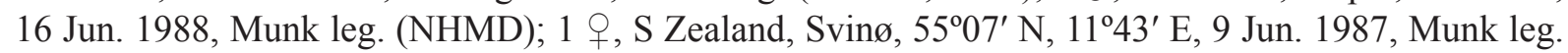
(NHMD).

FINLAND: 1 q, EH. Lammi, 6772: 391, 15 Jul. 1976, Koponen leg. (NHMD).

GERMANY: 1 ðૈ, Dransfeld, 15 May 1966, Haeselbarth leg. (ZSSM); 2 qq, 1 ð, Glonn, 18 Aug. 1970, swept on blueberry, Haeselbarth leg. (ZSSM).

ITALY: 1 đ, South Tyrol, Kaltern, 500 m, 22 Sep. 1978, Haeselbarth leg. (ZSSM); 1 đ, South Tyrol, Merano, 700 m, 22 Jul. 1966, Haeselbarth leg. (ZSSM); 2 qq , South Tyrol, Parcines, 850 m, 13 Jul. 1966 and 1 Sep. 1967, Haeselbarth leg. (ZSSM, ZISP); 1 \&, South Tyrol, Riva Rocchetta, 400-800 m, 20 May 1982, Haeselbarth leg. (ZSSM).

\section{Distribution}

Palaearctic Region: Denmark (first record), Finland (first record), Germany (first record), Italy (first record), and Russia (Yu et al. 2012).

\section{Material examined}

*Aspilota vernalis Stelfox \& Graham, 1951

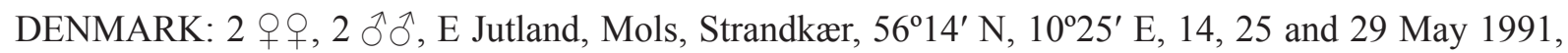
Munk leg.

\section{Distribution}

Western Palaearctic (Yu et al. 2012). Denmark (first record).

Genus Chasmodon Haliday, 1838

Published material

Chasmodon apterus (Nees, 1812)

"Resengaard and Laasby (E Jutland)" (Nielsen 1994).

\section{Distribution}

Palaearctic region (Yu et al. 2012). 
Genus Dinotrema Forster, 1863

Published material

Dinotrema acompressum Munk \& Peris-Felipo, 2014

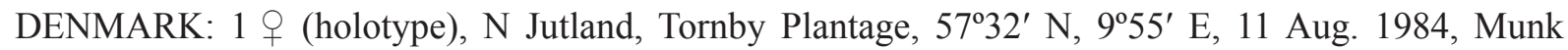
leg. (NHMW); 1 ( (paratype), E Jutland, Klattrup, S of Vejle, 2 Jun. 1983, Munk leg. (NHMW); 1 ㅇ (paratype), E Jutland, Højen Bæk, 5 km S of Vejle, 9 Jun. 1986, Munk leg. (NHMW); 1 (paratype), E Jutland, Frisenborg, 56 $16^{\prime}$ N, 9 $9^{\circ} 54^{\prime}$ E, 28 Aug. 1986, Munk leg. (ENV); 2 q 9 (paratypes), N Jutland, Villestrup, 56 $46^{\prime}$ N, $9^{\circ} 56^{\prime}$ E, 14 Jun. 1984, Munk leg. (ENV, ZISP); 1 (paratype), N Jutland,

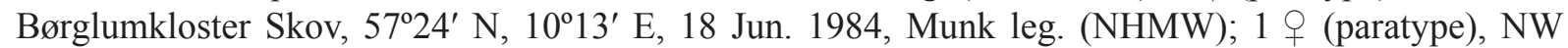
Jutland, Morup Mølle, 56 ${ }^{\circ} 51^{\prime}$ N, $8^{\circ} 22^{\prime}$ E, 15 Jun. 1985, Munk leg. (NHMW) (Peris-Felipo et al. 2014a).

\section{Distribution}

Western Palaearctic (Peris-Felipo et al. 2014a). Only known from Denmark.

\section{Published material}

Dinotrema agaricophagum Munk \& Peris-Felipo, 2013

DENMARK: 3 우 (holotype and paratypes), E Jutland, Mols Strandkær, 56 $6^{\circ} 14^{\prime}$ N, 10 $0^{\circ} 25^{\prime}$ E, 4 Oct. 1983, Munk leg. (ENV, NMA); 1 † (paratype), N Jutland, Åstrup Skov, NJ67, 16 Sep. 1982, Munk leg. (NHMW); 1 (paratype), N Jutland, NJ77, 20 Aug. 1985, Munk leg. (NHMW) (Peris-Felipo et al. 2014a).

\section{Distribution}

Western Palaearctic (Peris-Felipo et al. 2014a).

\section{Remarks}

Specimens were collected from Agaricus sp. (Fungi).

\section{Published material}

Dinotrema alysiae Munk \& Peris-Felipo, 2013

DENMARK: 3 우 (holotype and paratypes), E Jutland, Mols Strandkær, 56 ${ }^{\circ} 14^{\prime} \mathrm{N}, 10^{\circ} 25^{\prime}$ E, $30 \mathrm{Jul}$. and 2 Sep. 1991, Munk leg. (NMA); 2 q+ (paratypes), E Jutland, Yding Skov, SW of Skanderborg, 6

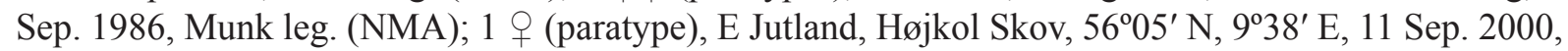
Munk leg. (ENV) (Peris-Felipo et al. 2014a).

\section{Distribution}

Western Palaearctic (Munk et al. 2013a; Peris-Felipo et al. 2014a).

\section{Published material}

Dinotrema amoenidens (Fischer, 1973)

DENMARK: 5 우, E Jutland, Klattrup, S of Vejle, NG36, 26-27 Sep. 1982, Munk leg. (NHMW); 7 우, N Jutland, NJ67, Slotved Skov, 21 Jun. 1982, Munk leg. (NHMW) (Peris-Felipo et al. 2014a).

\section{Distribution}

Palaearctic Region (Peris-Felipo et al. 2014a). 


\section{Published material}

Dinotrema areolatum (Stelfox \& Graham, 1950)

DENMARK: 1 + , E Jutland, Vingsted, W of Vejle, 8 Jun. 1983, Munk leg. (ENV) (Peris-Felipo et al. 2014a).

\section{Material examined.}

DENMARK: 1 +, E Jutland, Løverodde, 31 Jul. 1984, Munk leg.; 1 đે, N Jutland, NJ67, Åstrup Skov,

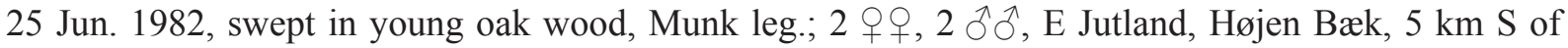
Vejle, 4 Jul. 1983, 6 Jul., 2 and 25 Aug. 1984, Munk leg.; 1 + , E Jutland, Nørreris Skov, $10 \mathrm{~km}$ of

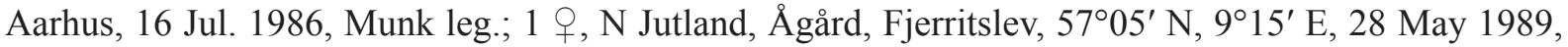
Munk leg.; 1 q, E Jutland, Tirsbæk, 17 Jul. 1983, Munk leg.

\section{Distribution}

Western Palaearctic (Peris-Felipo et al. 2014a).

\section{Remarks}

One specimen was collected in oak wood.

\section{Published material}

Dinotrema cahitum Munk \& Peris-Felipo, 2014

DENMARK: 1 ( holotype), E Jutland, Klattrup, 7 km S of Vejle, 19 Jul. 1983, Munk leg. (NMA); 1 ㅇ (paratype), E Jutland, Klattrup, S of Vejle, 29 Jul. 1978, Munk leg. (NHMW); 1 q (paratype), E Jutland, Tirsbæk E of Vejle, 20 Jul. 1983, Munk leg. (ENV); 1 q (paratype), W Jutland, Ammitsbøl Skov, 24 Jul. 1984, Munk leg. (NMA); 1 ㅇ (paratype), E Jutland, Højen Bæk, 5 km S of Vejle, 18 Jul. 1984, Munk leg. (ENV) (Peris-Felipo et al. 2014a).

\section{Distribution}

Western Palaearctic (Peris-Felipo et al. 2014a). Only known from Denmark.

\section{Published material}

Dinotrema collybiae Munk \& Peris-Felipo, 2014

DENMARK: 2 우 (holotype and paratype), E Jutland, Højen Bæk, $5 \mathrm{~km} \mathrm{~S}$ of Vejle, 15 Jun. 1989, Munk leg. (ENV, NMA) (Peris-Felipo et al. 2014a).

\section{Distribution}

Western Palaearctic (Peris-Felipo et al. 2014a). Only known from Denmark.

\section{Published material}

Dinotrema compressum (Haliday, 1838)

DENMARK: 1 q, E Jutland, Højen Bæk, 5 km S of Vejle, 21 Jun. 1986, Munk leg. (ENV) (Peris-Felipo et al. 2014a).

\section{Additional material examined}

DENMARK: 1 q, N Jutland, Åstrup Skov, 18 Jun. 1984, Munk leg.; 1 + , E Jutland, Klattrup Bygade., FB KP, 24-25 Aug. 1982, Munk leg.; 1 ㅇ, Funen, Smedsholm Skov, 3 km W of Fjelsted, 4 Jun. 1982, Munk leg.; 1 ㅇ, E Jutland, Højen Bæk, 24 May 1984, Munk leg.; 2 + , E Jutland, Højkol Skov,

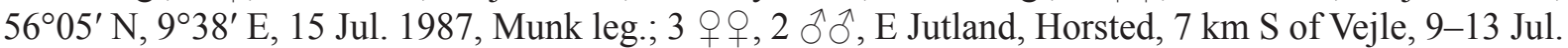
1977, Munk leg.; 1 ․ E Jutland, Nørreris Skov, 10 km NW of Aarhus, 20 Jun. 1985, Munk leg.; 1 , 
E Jutland, Tinskær Skov, S of Vejle, 20 Aug. 1998, Munk leg.; 3 q $ᄋ$, E Jutland, Yding Skov, 56 $00^{\prime}$ N, 948' E, 24 Jun. 1984 and 1 Jul. 1985, Munk leg.

\section{Distribution}

Western Palaearctic (Peris-Felipo et al. 2014a).

\section{Material examined}

*Dinotrema concinnum (Haliday, 1838)

DENMARK: 1 ㅇ, 1 đૈ, E Jutland, Højen Bæk, 5 km S of Vejle, 1 Jul. 1983, and 7 Jul. 1984, Munk leg.

\section{Distribution}

Palaearctic Region (Peris-Felipo et al. 2014a). Denmark (first record).

\section{Material examined}

*Dinotrema contracticorne (Fischer, 1974)

DENMARK: 1 q, E Jutland, Staksrode Skov, 10 Jun. 1984, Munk leg.

\section{Distribution}

Western Palaearctic (Peris-Felipo et al. 2014a). Denmark (first record).

\section{Published material}

Dinotrema costulatum (Thomson, 1895)

"Sondbg [=Søndeborg in South Jutland]" (Fischer 1972).

\section{Material examined}

DENMARK: 1 , , E Jutland, NG36, Børkop Skov, 1 Jul. 1982, Munk leg.; 1 q, E Jutland, Tirsbæk, E of Vejle, 20 Jul. 1983, Munk leg.; 2 우, E Jutland, Horsted, S of Vejle, 27-28 Aug. 1976, Munk leg.; 1 Õ, E Jutland, Assens at Mariager Fjord, 20 Aug. 1986, Munk leg.; 1 , E Jutland, Elsegårde Skov,

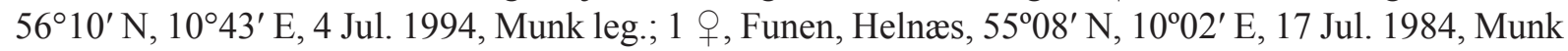
leg.; 3 우, 1 Ō. E Jutland, Klattrup, 7 km S of Vejle, 4 and 24-25 Aug. 1982 and 3 Jul. 1983, Munk leg.; 1 +, 1 §̂, E Jutland, Højen Bæk, 1 Jul. 1982 and 4 Aug.-7 Sep. 1986, Munk leg.; 1 q, E Jutland, Rohden, $12 \mathrm{~km}$ E of Vejle, 2 Jul. 2000, swept in wet deciduous wood, Munk leg.; 2 9 9 , E Jutland, Nim Skov, 26 Apr. and 26 Aug. 1984, Munk leg.; 1 ㅇ, 1 O , E Jutland, Skanderborg, Modervejen, 21 Sep. 1984, Munk leg.

\section{Distribution}

Western Palaearctic (Peris-Felipo et al. 2014a).

\section{Remarks}

One specimen was collected in wet deciduous wood.

\section{Material examined}

*Dinotrema cratocera (Thomson, 1895)

DENMARK: 1 + , N Jutland, Åstrup Skov, 19 Jun. 1984, Munk leg.; 1 q, N Jutland, Måstrup Mose,

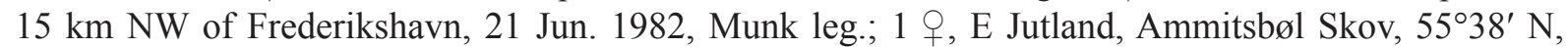
$9^{\circ} 26^{\prime}$ E, 16 Jul. 1982, Munk leg.; 2 우, E Jutland, Bjørnholm Skov, 56²0’ N, 1043' E, 7 Jul. 1989,

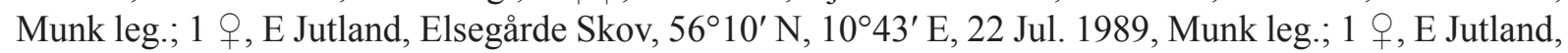

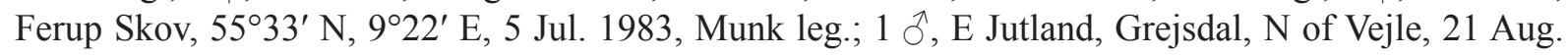


1984, Munk leg.; 2 q ㅇ, E Jutland, Hessel, S of Grenå, 56 $23^{\circ}{ }^{\prime}$ N, 10 $0^{\circ} 52^{\prime}$ E, 9 and 13 Aug. 1989, Munk

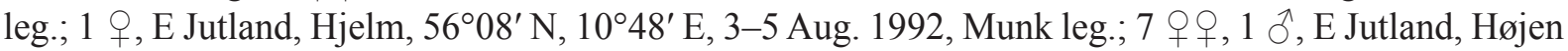
Bæk, S of Vejle, Sep.1981, 17 Jul. 1982, 1 Jun., 5, 20 and 30 Jul. 1984, 16 Jun. 1986, Munk leg.; 1 q, E

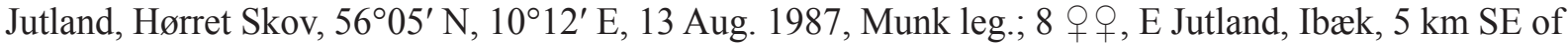
Vejle, 15 Jun. 1989, Munk leg.; 3 우, 1 ㄱ, E Jutland, Klattrup, 7 km S of Vejle, 15 Jul. 1982, 28 Jun. and 13 Jul. 1983, Munk leg.; 3 우, E Jutland, Pedersholms Allé, Vejle, 1 Jul. 1982, Munk leg.; 1 q, E Jutland, Rosenvold, 28 Jul. 1984, Munk leg.; 2 우오, E Jutland, Rugård Sønderskov, 56 $16^{\prime}$ N, 1049' E, 20 Jul. 1986, Munk leg.; 3 우오, E Jutland, Yding Skov, 56 $00^{\prime}$ N, 948' E, 4 Jul. and 24 Jun. 1985,

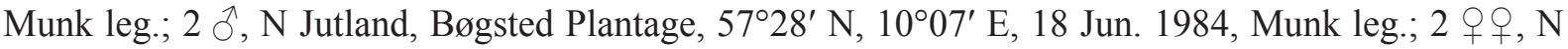
Jutland, Frederikshavn, Vandværksskoven, 2 Aug. 1987, Munk leg.; 2 우, N Jutland, Tornby Plantage,

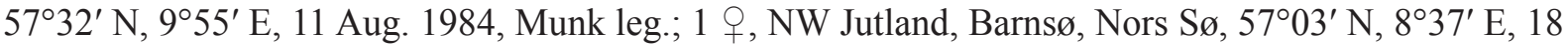
Aug. 1989, Munk leg.; 1 q, NW Jutland, Mønsted Skov, 17 Jun. 1984, Munk leg.; 1 , , N Jutland, St. Vildmose, 6 Aug. 1984, Munk leg.; 1 ㅇ, N Jutland, St. Vildmose, 6 Aug. 1984, Munk leg.; 1 ㅇ, 1 ○े, E Jutland, Viuf Skov, 21 Jun. 1986, Munk leg.

\section{Distribution}

Palaearctic Region (Peris-Felipo et al. 2014a). Denmark (first record).

\section{Material examined}

*Dinotrema cruciatum (Fischer, 1973)

DENMARK: 1 +, E Jutland, Højen Bæk, 10 Jul. 1984, Munk leg.

\section{Distribution}

Palaearctic Region (Peris-Felipo et al. 2014a). Denmark (first record).

\section{Published material}

Dinotrema cruciforme (Fischer, 1973)

DENMARK: 1 , E Jutland, Højen Bæk, 5 km S of Vejle, 23 Aug. 1984, Munk leg. (NMA) (PerisFelipo et al. 2014a).

\section{Additional material examined}

DENMARK: 3 우, 1 ㄱ, E Jutland, Klattrup, $10 \mathrm{~km} \mathrm{~S}$ of Vejle, 12 Jul. 1981, 26 Jul. 1982, 22 and 31 Aug. 1982, Munk leg.; 1 ㅇ, E Jutland, Horsted, S of Vejle, 2 Nov. 1978, Munk leg.; 1 , , E Jutland, Randbøldal, W of Vejle, 7 Jun. 1983, Munk leg.; 1 中, E Jutland, Højen Bæk, 5 km S of Vejle, 23 Aug. 1984, Munk leg.

\section{Distribution}

Palaearctic Region (Peris-Felipo et al. 2014a).

\section{Published material}

Dinotrema curtisetum Munk \& Peris-Felipo, 2013

DENMARK: 1 q (holotype), 1 q (paratype), E Jutland, Hessel, S of Grenå, 56 23' N, 10 $52^{\prime}$ E, 9 Aug. and 13 Aug. 1989, Munk leg. (ENV, NMA); 1 \& (paratype), S Jutland, Klelund Plantage, 23 Aug. 1986, Munk leg. (NMA) (Peris-Felipo et al. 2014a).

\section{Distribution}

Western Palaearctic (Munk et al. 2013b; Peris-Felipo et al. 2014a). Only known from Denmark. 


\section{Material examined}

*Dinotrema denticulatum (Stelfox \& Graham, 1951)

DENMARK: 1 + , E Jutland, Klattrup Skov, S of Vejle, 10 Sep. 1982, Munk leg.; 1 , E Jutland, Yding Skov, SW of Skanderborg, 6 Sep. 1986, Munk leg.; 1 q, E Jutland, Højkol Skov, 56 $05^{\prime}$ N, $9^{\circ} 38^{\prime}$ E, 14 Sep. 1987, Munk leg.; 1 +, E Jutland, Mols, Strandkær, 56²14' N, 10²5’ E, 2 Sep. 1991, Munk leg.

\section{Distribution}

Western Palaearctic (Peris-Felipo et al. 2014a). Denmark (first record).

\section{Material examined}

*Dinotrema (Leptotrema) dentifemur (Stelfox, 1943)

DENMARK: 1 + , E Jutland, Klattrup, S of Vejle, NG 36, 10 Sep. 1982, Munk leg.; 1 ô, SW Jutland, outside of the sea dike, $1 \mathrm{~km} \mathrm{~S}$ of the Rømø Dam, 31 May 1982, Munk leg.; 1 \&, E Jutland, Egå, $10 \mathrm{~km}$ NE of Aarhus, 13-19 Sep. 1984, Munk leg.; 1 P, E Jutland, Frisenborg, 56 $6^{\circ} 16^{\prime}$ N, 9 ${ }^{\circ} 54^{\prime}$ E, 28 Jul. 1986,

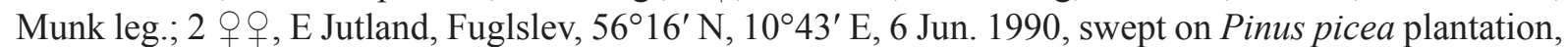
and 10 Nov. 2000, Munk leg.; 1 ㅇ, E Jutland, Højkol Skov, 56 $05^{\prime}$ N, $9^{\circ} 38^{\prime}$ E, 7 Jul. 1986, Munk leg.;

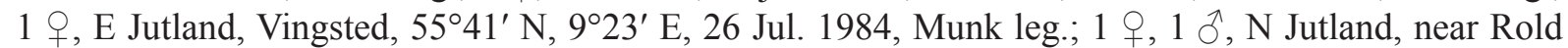

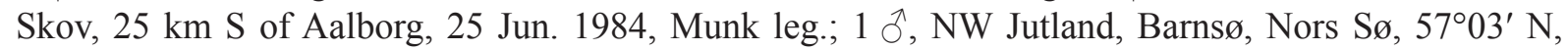
$8^{\circ} 37^{\prime}$ E, 18 Aug. 1989, Munk leg.

\section{Distribution}

Palaearctic Region (Yu et al. 2012). Denmark (first record).

\section{Remarks}

One specimen was swept in a Pinus picea L. plantation.

\section{Published material}

Dinotrema deprane Munk \& Peris-Felipo, 2013

DENMARK: 1 (holotype), 13 q+ (paratypes), E Jutland, Højen Bæk, 1 Jul. 1982, 6 Jun. 1983, 12 Jun., 7 Jul. and 19 Sep. 1984, 16 Jun. 1986 (NMA) (Peris-Felipo et al. 2014a).

\section{Distribution}

Western Palaearctic (Munk et al. 2013b; Peris-Felipo et al. 2014a). Only known from Denmark.

\section{Published material}

Dinotrema digitatum Munk \& Peris-Felipo, 2014

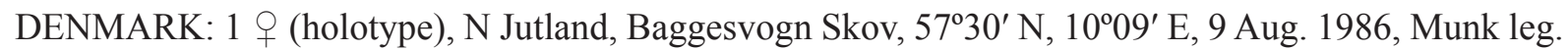
(NMA); 2 $q$ (paratypes), E Jutland, Højen Bæk, 5 km S of Vejle, 6 Jul. 1984, Munk leg. (NHMW); 6 우오 (paratypes), N Jutland, Villestrup, 56 $6^{\circ} 46^{\prime}$ N, 9 9 56' E, 25-26 Jun. 2984, Munk leg. (NHMW, ZISP);

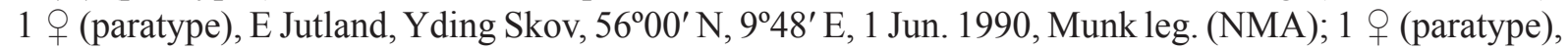

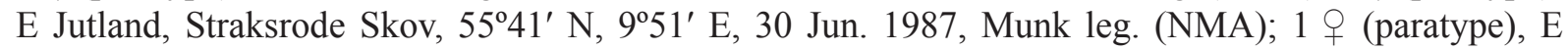
Jutland, Tirsbæk, 5 km E of Vejle, 29 Jul. 1984, Munk leg. (NMA); 1 \& (paratype), E Jutland, Klattrup, S of Vejle, 27 Aug. 1982, Munk leg. (ENV); 1 q (paratype), N Jutland, Villestrup, 56 $46^{\prime}$ N, $9^{\circ} 56^{\prime}$ E, 18 Jun. 1984, Munk leg. (ENV) (Peris-Felipo et al. 2014a).

\section{Distribution}

Western Palaearctic (Peris-Felipo et al. 2014a). Only known from Denmark. 


\section{Published material}

$$
\text { Dinotrema dimidiatum (Thomson, 1895) }
$$

"Oberlehrer Wüstnei" (Thomson 1895), "Ile d'Alsen, trouvé par Wüstnei" (Marshall 1900).

\section{Additional material examined}

DENMARK: 1 + , E Jutland, Bindeballe, 20 May 1984, Munk leg.; 1 q, E Jutland, Sillerup, 12 km SW of Silkeborg, 29 Apr. 1987, Munk leg.; 3 웅, 4 소, E Jutland, Klattrup Skov, 10 km S of Vejle, 2 May

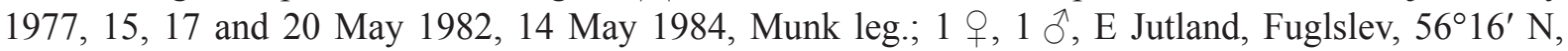

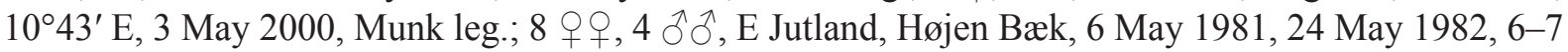
and 20 May 1983, 6, 10 and 21 May 1984, 19 May 1985, and 9 May 1987, Munk leg.; 1 +, E Jutland, Nørreris Skov, W of Aarhus, 8 May 1985, Munk leg.; 1 +, E Jutland, Skærsø, PH03, Kælderskov, 25 May 1996 and 30 Apr. 1997, Munk leg.; 1 đ̃. E Jutland, Volstrup Skov, 56²0' N, 958' E, 11 May

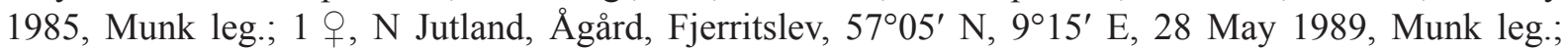

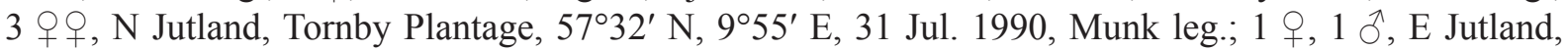
Frisenborg, 17 May 1984, Munk leg.; 1 ठ̂, E Jutland, Kjellerup, 15 May 1986, Munk leg.; 1 ㅇ, NE Zealand, Hareskoven v. Frederiksdal, 7 May 1976, Munk leg.

\section{Distribution}

Palaearctic Region (Peris-Felipo et al. 2014a).

\section{Material examined}

*Dinotrema divisum (Stelfox \& Graham, 1950)

DENMARK: 1 + , E Jutland, Yding Skov, SW of Skanderborg, 6 Sep. 1986, Munk leg.; 1 ô, N Jutland, Skårupgård Skov, 22 Jun. 1982, swept in Pinus wood, Munk leg.; 1 + , S Jutland, Arild, 1 Jul. 1986, Munk leg.; 1 ㅇ, N Jutland, Rold Skov, Mossø, 14 Aug. 1984, Munk leg.; 1 + , N Jutland, Slotved Skov, 13-14 Jun. 1982, Munk leg.; 1 + , Funen, Uggerslev Plantage, 24 Jul. 1985, Munk leg.; 1 +, E Jutland,

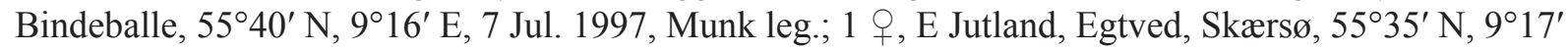
E, 13 Jul. 1985, Munk leg.; 1 + , E Jutland, Nørreris Skov, 10 km NW of Aarhus, 16 Jul. 1986, Munk

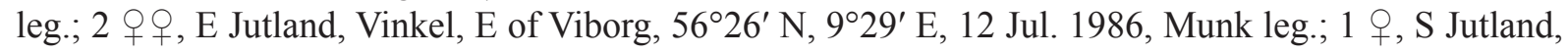

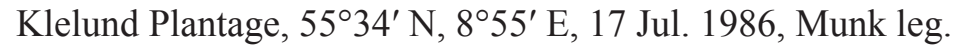

\section{Distribution}

Palaearctic Region (Peris-Felipo et al. 2014a). Denmark (first record).

\section{Remarks}

One specimen was swept in a Pinus wood.

\section{Published material}

\section{Dinotrema erythropum Forster, 1863}

DENMARK: 1 q, E Jutland, Frisenborg, 28 Jul. 1986, Munk leg. (NMA) (Peris-Felipo et al. 2014a).

\section{Additional material examined}

DENMARK: 1 ð̊, NE Zealand, Copenhagen, Utterslev Mose, 8 Jun. 1976, Munk leg.; 1 q, E Jutland, Frisenborg, 28 Jul. 1986, Munk leg.; 1 +, E Jutland, Ammitsbøl Skov, 55³8' N, 9²6' E, 16 Jul. 1982,

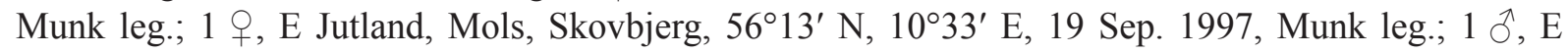
Jutland, Yding Skov, $56^{\circ} 00^{\prime}$ N, $9^{\circ} 48^{\prime}$ E, 9 Aug. 1985, Munk leg. 


\section{Distribution}

Western Palaearctic (Peris-Felipo et al. 2014a).

\section{Published material}

Dinotrema falsificum (Stelfox \& Graham, 1950)

DENMARK: 1 , , N Jutland, NJ 67, Baggesvogn Skov, 24 Jun. 1994, Munk leg. (ENV) (Peris-Felipo et al. 2014a).

\section{Additional material examined}

DENMARK: 1 + , E Jutland, Aarhus, Trondhjemsgade, 6 Sep. 1986, Munk leg.; 1 q, N Jutland, Tvilho, 23 Aug. 1986, Munk leg.; 2 우, E Jutland, Assens at Mariager Fjord, 20 Aug. 1986, Munk leg.

\section{Distribution}

Western Palaearctic (Peris-Felipo et al. 2014a).

\section{Published material}

Dinotrema fulvicorne (Haliday, 1838)

DENMARK: 1 + , N Jutland, Højen Bæk, 5 km S of Vejle, 4 Jun. 1989, Munk leg. (ENV) (Peris-Felipo et al. 2014a).

\section{Additional material examined}

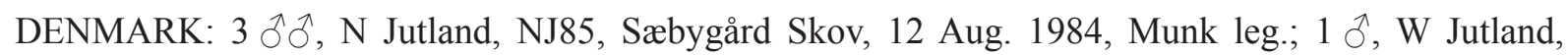
Nørholm Skov, 8 Jul. 1984, Munk leg.; 1 đૈ, E Jutland, Djursland, Jun. 1984, Munk leg.; 1 ô, E Jutland,

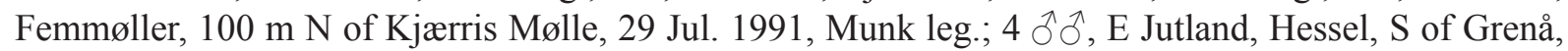

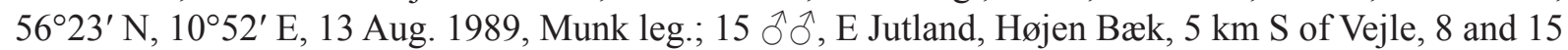
Jul. 1982, 22 and 30 Jul. 1983, 6, 13-14, 18, 23, 30 Jul., 2, 18, 20, 23-24 Aug. 1984, Munk leg.; 1 9, E Jutland, Klattrup, S of Vejle, 13 Jul. 1982, Munk leg.; 1 Oे, E Jutland, Nørreskov, 10 km E of Kolding,

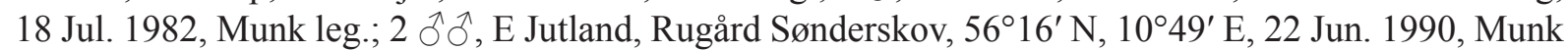
leg.; 2 ठึ⿱一兀), E Jutland, Yding skov, SW of Skanderborg, 6 Sep. 1986, Munk leg.

\section{Distribution}

Western Palaearctic (Peris-Felipo et al. 2014a).

\section{Published material}

Dinotrema glabrideum Munk \& Peris-Felipo, 2014

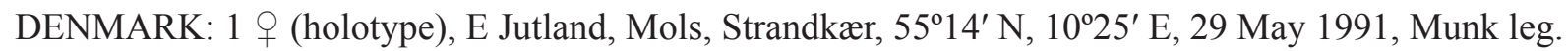
(NMHW); 1 q (paratype), E Jutland, Højen Bæk, 5 km S of Vejle, 18 Jul. 1984, Munk leg. (ENV); 2 우 (paratypes), E Jutland, Højen Bæk, 24 May 1984, Munk leg. (ENV; NMA) (Peris-Felipo et al. 2014a).

\section{Distribution}

Western Palaearctic (Peris-Felipo et al. 2014a). Only known from Denmark.

\section{Published material}

Dinotrema glabrum (Stelfox \& Graham 1951)

DENMARK: 2 우, E Jutland, Højkol Skov, 56 $05^{\prime}$ N, 9³8' E, 16 Jul. and 22 Aug. 1987, Munk leg. (ENV) (Peris-Felipo et al. 2014a). 


\section{Additional material examined}

DENMARK: 1 + , W Jutland, Bredsgårde, 10 km W of Viborg, 12 Jul. 1986, Munk leg.; 1 +, N Jutland, Baggesvogn, 20 Jun. 1984, Munk leg.; 1 , , W Jutland, Haslund, 2 Jul. 1984, Munk leg.; 2 q + , E Jutland, Salten Skov, SE of Silkeborg, 7 Jul. 1986, Munk leg.; 3 q , E Jutland, Højen Bæk, 5 km S of Vejle, 15 Jul. 1982 and 2 Aug. 1984, Munk leg.; 2 우, E Jutland, Hørret Skov, 56 05' N, 10¹2' E, 7 Jul. 1986 and 7 Aug. 1987, Munk leg.; 1 q, E Jutland, Klattrup, 7 km S of Vejle, 4 Aug. 1983, Munk leg.; 1 q, E Jutland, Mols, Skovbjerg, 56 ${ }^{\circ} 13^{\prime}$ N , 10³3' E, 11 Sep. 1997, Munk leg.; 3 q $ᄋ$, E Jutland, Nørreris Skov, 10 km NW of Aarhus, 20 Jun. and 14 Jul. 1985, 5 Jul. 1987, Munk leg.; 1 ô, E Jutland, Nørreskov,

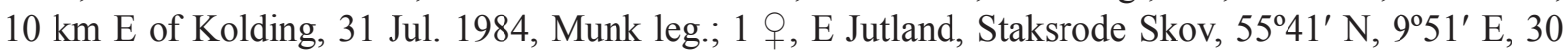

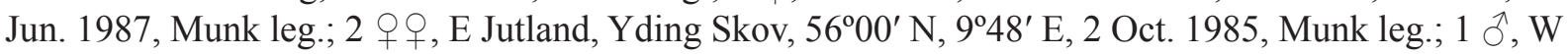

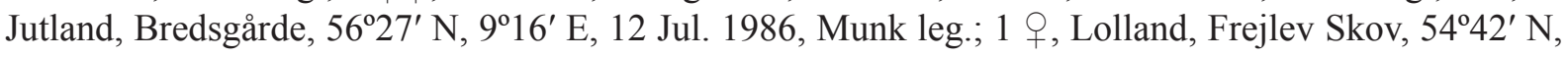
$11^{\circ} 51^{\prime}$ E, 9 Jun. 1987, Munk leg.

\section{Distribution}

Palaearctic Region (Peris-Felipo et al. 2014a).

\section{Published material}

Dinotrema haeselbarthi Munk \& Peris-Felipo, 2013

DENMARK: 1 ( (paratype), E Jutland, Yding Skov, 56 $00^{\prime}$ N, 9 $9^{\circ} 48^{\prime}$ E, 24 May 1985, Munk leg.

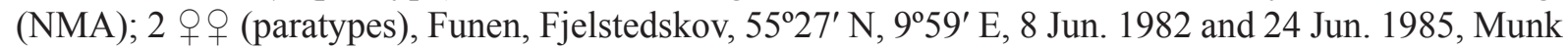
leg. (NMA); 1 (paratype), E Jutland, Højen Bæk, 5 km S of Vejle, 7 Jul. 1984, Munk leg. (ENV) (Peris-Felipo et al. 2014a).

\section{Distribution}

Western Palaearctic (Munk et al. 2013b; Peris-Felipo et al. 2014a).

\section{Published material}

Dinotrema helote Munk \& Peris-Felipo, 2014

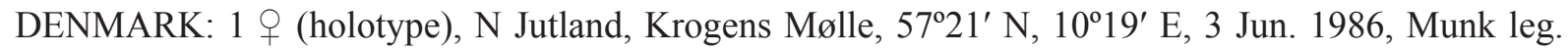
(NHMW); 1 q (paratype), E Jutland, Elsegårde Skov, 56 20' N, 1043' E, 7 Jul. 1988, Munk leg. (ENV); 1 (paratype), N Jutland, Øster Vrå, $30 \mathrm{~km} \mathrm{SW}$ Frederikshavn, swept on Paludella sp., 30 May 1986,

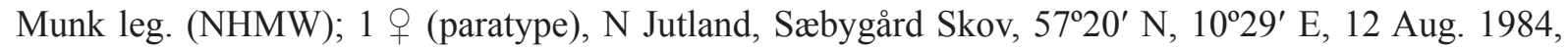

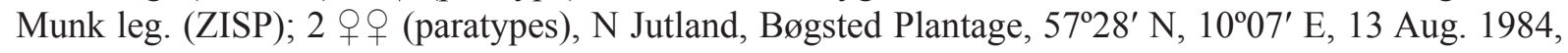
Munk leg. (ENV, NMA) (Peris-Felipo et al. 2014a).

\section{Distribution}

Western Palaearctic (Munk et al. 2013b; Peris-Felipo et al. 2014a). Only known from Denmark.

\section{Remarks}

One specimen swept from moss, Paludella sp.

\section{Material examined}

*Dinotrema hodiense (Fischer, 1976)

DENMARK: 1 q, S Jutland, Askelund, 15 Aug. 1991, Munk leg.; 1 \&, E Jutland, Krattet, 19 Aug. 1991, Munk leg. (Peris-Felipo et al. 2014a).

\section{Distribution}

Palaearctic Region (Peris-Felipo et al. 2014a). Denmark (first record). 


\section{Published material}

Dinotrema jaculans (Haliday, 1838)

DENMARK: 1 + , E Jutland, Rugård, Sønderskov, 56 $16^{\prime}$ N, 1049’ E, 22 Jun. 1990, Munk leg. (ENV) (Peris-Felipo et al. 2014a).

\section{Additional material examined}

DENMARK: 1 \&, N Jutland, Åsted, 16 Jun. 1984, Munk leg.; 1 \&, N Jutland, Åstrup, 18 Jun. 1984, Munk leg.; 1 ․ E Jutland, Grejsdal, N of Vejle, 23 Aug. 1987, Munk leg.; 5 우, E Jutland, Højen Bæk, 5 km S of Vejle, 27 Jun., 5-6, 11 and 23 Jul. 1984, Munk leg.; 1 9, E Jutland, Klattrup, 19 Jun. 1984,

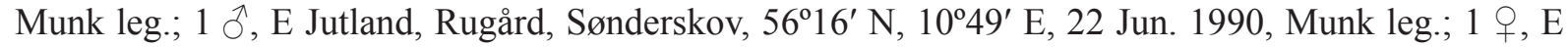

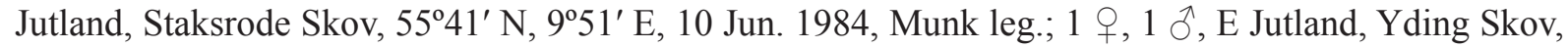
$56^{\circ} 00^{\prime} \mathrm{N}, 9^{\circ} 48^{\prime}$ E, 1 Jun. 1990, Munk leg.; 1 \%, NE Zealand, Copenhagen, Utterslev Mose, 8 Jun. 1976,

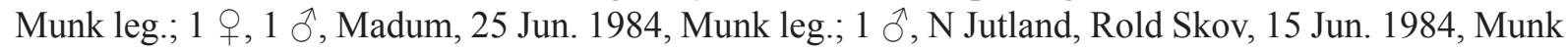
leg.; 1 9, N Jutland, Sindal, 22 Jul. 1985, Munk leg.

\section{Distribution}

Western Palaearctic (Peris-Felipo et al. 2014a).

\section{Material examined}

*Dinotrema latifemur (Fischer, 1975)

DENMARK: 1 क, E Jutland, Klattrup, 12 Jun. 1983, Munk leg.

\section{Distribution}

Palaearctic Region (Peris-Felipo et al. 2014a). Denmark (first record).

\section{Published material}

Dinotrema lepiotae Munk \& Peris-Felipo, 2014

DENMARK: 1 (holotype), N Jutland, NJ 67, Åstrup Skov, swept on Lepiota rhacodes, 16 Sep. 1982, Munk leg. (NHMW); 2 우 (paratypes), N Jutland, NJ 77, Sindal, swept on Lepiota procera, 19 Aug. 1985, Munk leg. (NHMW); 1 ㅇ (paratype), E Jutland, Klattrup, S of Vejle, 29 Aug. 1982, Munk leg. (ENV); 4 + $९$ (paratypes), E Jutland, Mols Strandkær, 56 ${ }^{\circ} 14^{\prime}$ N, $10^{\circ} 25^{\prime}$ E, 4 Oct. 1983, Munk leg. (ENV; ZISP; NMA) (Peris-Felipo et al. 2014a).

\section{Distribution}

Western Palaearctic (Peris-Felipo et al. 2014a). Only known from Denmark.

\section{Remarks}

One specimen swept from Chlorophyllum rhacodes (Vittad.) Vellinga and two specimens from Macrolepiota procera (Scop.) Singer (Fungi).

\section{Published material}

Dinotrema lobatum Munk \& Peris-Felipo, 2013

DENMARK: 1 (holotype), E Jutland, Højen Bæk, 5 km S of Vejle, 23 Jul. 1984, Munk leg. (NHMW); 2 우 (paratypes), N Jutland, NJ77, Sindal, 22 Jul. 1985, Munk leg. (NHMW, NMA); 1 (paratype), N Jutland, near Rold Skov, 25 km S of Aalborg, 27 Jul. 1985, Munk leg. (NHMW) (Peris-Felipo et al. 2014a). 


\section{Distribution}

Western Palaearctic (Munk et al. 2013b; Peris-Felipo et al. 2014a).

\section{Published material}

Dinotrema longicarinatum (Fischer, 1976)

DENMARK: 1 , , E Jutland, Molslaboratotiet, 56 ${ }^{\circ} 14^{\prime} \mathrm{N}, 10^{\circ} 25^{\prime} \mathrm{E}$, Munk leg. (NHMW) (Peris-Felipo et al. 2014a).

\section{Distribution}

Western Palaearctic (Peris-Felipo et al. 2014a).

\section{Published material}

Dinotrema macrocera (Thomson, 1895)

DENMARK: 1 + , E Jutland, Højen Bæk, 5 km S of Vejle, 23 Aug. 1984, Munk leg. (ENV) (Peris-Felipo et al. 2014a).

\section{Additional material examined}

DENMARK: 2 우, N Jutland, Åstrup Skov, 19 Jun. 1984, Munk leg.; 1 q, E Jutland, Børkop, 1 Jul.

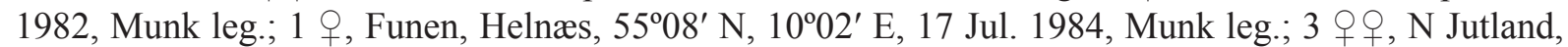
Haraldskær, 26 Aug. 1984, Munk leg.; 1 9, E Jutland, Jelling Skov, 25 Sep. 1983, Munk leg.; 1 J , E Jutland, Mols, Strandkær, 29 Sep. 1986, Munk leg.; 1 ㅇ, E Jutland, Rosenvold, N of Vejle, NG 57, 28 Jul. 1984, Munk leg.; 12 우, E Jutland, Tirsbæk, 5 km E of Vejle, 18 Aug. 1984 and 16 Jun. 1986, Munk leg.; 1 ㅇ, E Jutland, Tiufkær Skov, 30 Jun. 1983, Munk leg.; 1 ㅇ, 2 ふૈ ô, E Jutland, Viuf Skov, S of Vejle, 24 and 29 Aug. 1984, Munk leg.; 1 ․, E Jutland, Randbøldal, W of Vejle, 7 Jun. 1983, Munk leg.; 3 우, N Jutland, Sæbygård Skov, 12 Aug. 1984, Munk leg.; 1 ․, N Jutland, Sæbygård Skov, NJ 85, 12 Aug. 1984, Munk leg.; 2 우, N Jutland, Tværsted Plantage, NJ 78, 17 and 20 Jun. 1982, Munk leg.; 6 우, N Jutland, Uggerby Plantage, 57²35' N, 1005' E, 22 Jun. 1983, 10 Aug. 1984 and 24 Jul. 1985, Munk leg.; 1 , , W Jutland, Grene, S of Billund, 3 Jul. 1984, Munk leg.; 3 우, , W Jutland, Haslung, NE of Ribe, 2 Jul. 1984, Munk leg.; 1 क, W Jutland, Tvilho, 30 km NE of Esbjerg, 17 Jul. 1986, Munk leg.; 1 ,

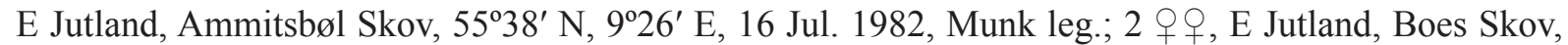
$56^{\circ} 03^{\prime}$ N, $9^{\circ} 50^{\prime}$ E, 24 Jun. 1985, Munk leg.; 2 오, 1 ㅊ‥ E Jutland, Elsted Skov, 10 km N of Aarhus, 1

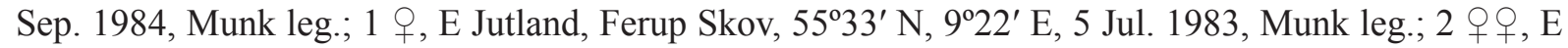
Jutland, Fuglslev Plantage, 56 $6^{\circ} 16^{\prime}$ N, 10 $0^{\circ} 43^{\prime}$ E, 2 Oct. 1988, Munk leg.; 1 + , E Jutland, Grejsdal, N of Vejle, 9 Jun. 1986, Munk leg.; 1 9, E Jutland, Grejsdal, 1 km NE of Lerbæk Mølle, 21 Aug. 1984, Munk

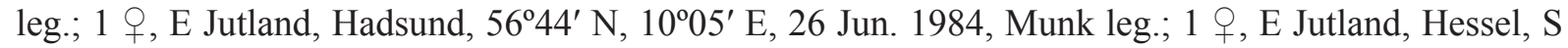

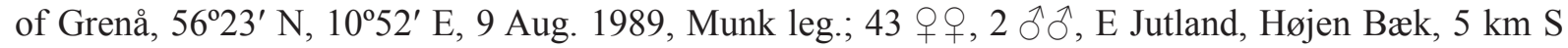
of Vejle, 8 Jul. 1982, 22 May and 12 Sep. 1983, 27 Jun., 5, 6 and 23 Jul., 17, 20, 23-25 and 30 Aug., 16 Sep. 1984, 9 Apr., 21 Jun., 24 Aug. and 7 Sep. 1986, 3 and 10 Jul. 1987, 4 Jun. 1989, Munk leg.; 4 우으, E Jutland, Højkol Skov, 56 05' N, 9³8' E, 7 Jul. 1986, 16 and 22 Aug. and 17 Sep. 1987, 21 Jul. 1988, Munk leg.; 3 우, E Jutland, Klattrup, 7 km S of Vejle, 2 Sep. 1983 and 16 Sep. 1984, Munk leg.; 15 q 9 , E Jutland, Nørreris Skov, 10 km NW of Aarhus, 14 Jul., 28 Aug., 5 and 11 Sep., 22 Oct. 1985, 16 Jul. and 4 Sep. 1986, Munk leg.; 1 , E Jutland, Nørreris, 10 km E of Kolding, 18 Jul. 1982, Munk leg.; 1 ㅇ, E Jutland, Palsgård Skov, 5601' N, 9²5' E, 28 Sep. 1987, Munk leg.; 2 우, E Jutland, Rugård Sønderskov, 56 $16^{\prime}$ N, 1049' E, 22 Jun. 1990, Munk leg.; 1 q, E Jutland, Skibet, W of Vejle,

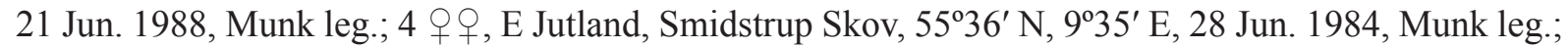

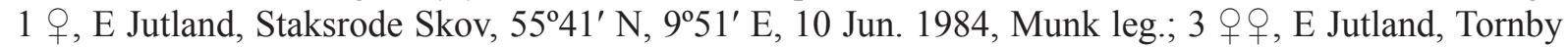

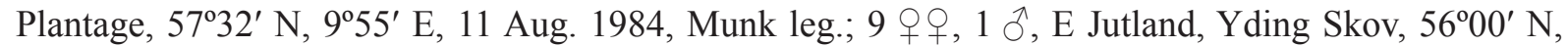
$9^{\circ} 48^{\prime}$ E, 24 Jun. 1984, 24 and 28 Jun., 1 Jul., 19 Sep..and 2 Oct. 1985, 1 Jun. 1990, Munk leg.; 1 9, N

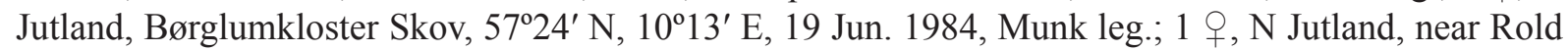


Skov, 25 km S of Alborg, 27 Jul. 1985, Munk leg.; 1 +, N Jutland, Sæbygård Skov, 57²0' N, 10²9' E, 3 Jun. 1986, Munk leg.; 1 , N Jutland, Villestrup, 56²46’ N, 956' E, 26 Jun. 1984, Munk leg.; 3 우, NW

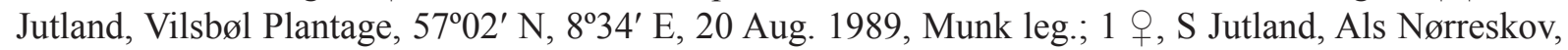

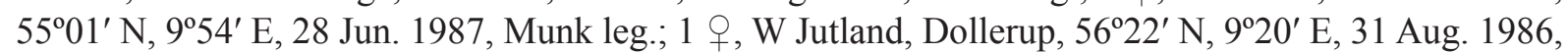

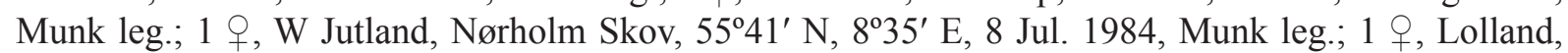

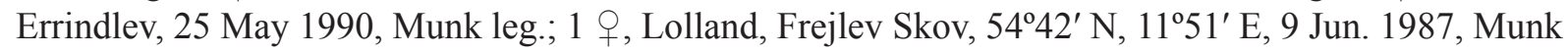
leg.; 1 ふै, N Jutland, Madum, 16 Aug. 1985, Munk leg.; 1 中, N Jutland, Rold, Mossø, 14 Aug. 1984, Munk leg.; 4 우, E Jutland, Smidstrup Skov, 28 Jun. 1984, Munk leg.; 1 Oૈ, N Jutland, St. Vildmose, 6 Aug. 1984, Munk leg.

\section{Distribution}

Palaearctic Region (Peris-Felipo et al. 2014a).

\section{Material examined}

*Dinotrema mesocaudatum van Achterberg, 1988

DENMARK: 2 우, Klattrup, 19 Aug. 1991, Munk leg.

\section{Distribution}

Palaearctic Region (Peris-Felipo et al. 2014a). Denmark (first record).

\section{Material examined}

*Dinotrema microcera (Thomson, 1895)

DENMARK: 1 , , E Jutland, Grejsdal, Verbæk, 9 Aug. 1985, Munk leg.; 5 q , E Jutland, Egå Skov,

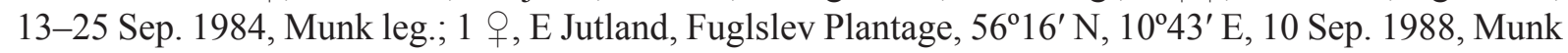

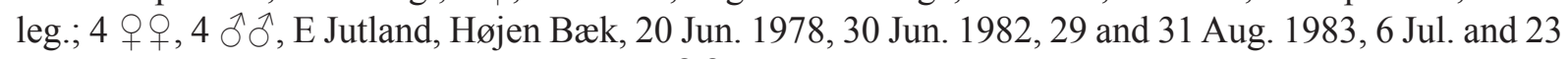
Aug. 1984, 10-11 Aug. 1985, Munk leg.; 2 우, E Jutland, Nørreris Skov, 10 km NW of Aarhus, 5 Oct.

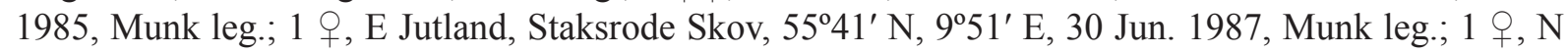
Jutland, Frederikshavn, Vandværksskoven, 2 Aug. 1987, Munk leg.; 1 ô, W Jutland, Klelund Plantage,

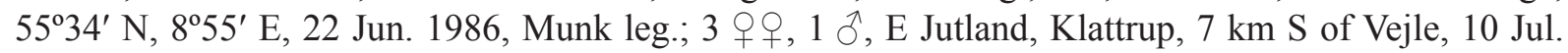
and 27 Aug. 1982, 4 Aug. 1983, and 1 Sep. 1984, Munk leg.; 1 q, N Jutland, Måstrup Mose, 15 km NW of Frederikshavn, 21 Jun. 1982, Munk leg.; 1 \&, 1 ô, W Jutland, Ammitsbøl Skov, 24 Jul. 1984 and 14 Aug. 1997, Munk leg.; 1 §ै, E Jutland, Monstrup, 21 Jun. 1982, Munk leg.; 1 ㅇ, E Jutland, Nim Skov, 26 Aug. 1984, Munk leg.; 1 \&, E Jutland, Skanderborg, highway, 21 Sep. 1984, Munk leg.; 1 đૈ, E Jutland, Smidstrup Skov, 28 Jun. 1984, Munk leg.; 1 \&, E Jutland, Strandkær Mols, 3 Oct. 1984, Munk

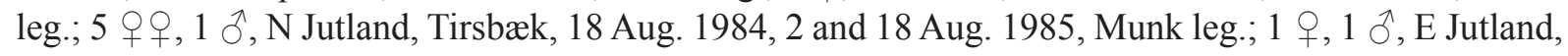
Tiufkær Skov, 20 Jul. 1984, Munk leg.; 1 只, E Jutland, Viuf Skov, 29 Aug. 1984, Munk leg.

\section{Distribution}

Western Palaearctic (Peris-Felipo et al. 2014a). Denmark (first record).

\section{Published material}

Dinotrema necrophilum (Hedqvist, 1972)

DENMARK: 3 우, E Jutland, Fuglslev, 56 ${ }^{\circ} 16^{\prime}$ N, 1043’ E, 26 May 1999, Munk leg. (ENV) (PerisFelipo et al. 2014a).

\section{Additional material examined}

DENMARK: 1 ㅇ, 2 ふో , E Jutland, Klattrup, $7 \mathrm{~km} \mathrm{~S}$ of Vejle, 12 Aug. 1982, 25 and 31 May 1983, Munk leg.; 10 우, E Jutland, Fuglslev, Stokbæk, 56¹6’ N, 1043’ E, 26 May 1999, Munk leg. 


\section{Distribution}

Western Palaearctic (Peris-Felipo et al. 2014a).

\section{Published material}

Dinotrema nervosum (Haliday, 1833)

Denmark (Yu et al. 2012).

\section{Distribution}

Palaearctic Region (Peris-Felipo et al. 2014a).

\section{Remarks}

According to Yu et al. (2012), D. nervosum was recorded from Denmark by Fischer (1972: 422). However, in Fischer's paper only a single nation (Ireland) is listed and, therefore, the presence of $D$. nervosum in Denmark is not confirmed.

\section{Material examined}

*Dinotrema nigricorne (Thomson, 1985)

DENMARK: 1 + , E Jutland, Klattrup, S of Vejle, 13 Jul. 1982, Munk leg.

\section{Distribution}

Palaearctic Region (Peris-Felipo et al. 2014a). Denmark (first record).

\section{Material examined}

*Dinotrema oleraceum (Tobias, 1962)

DENMARK: 5 우, 4 ふึ઼, E Jutland, Højen Bæk, 17 Aug. and 17-26 Sep. 1980, ex Amanita subescens (Pers. ex Fr.) Gray, Munk leg.

\section{Distribution}

Palaearctic Region (Peris-Felipo et al. 2014a). Denmark (first record).

\section{Remarks}

Collected from Amanita subescens (Pers. ex Fr.) Gray (Fungi).

\section{Published material}

Dinotrema occipitale (Fischer, 1973)

DENMARK: 1 ㅇ, N Jutland, Tornby Klitplantage, 57³2' N, 955' E, 31 Jul. 1990, Munk leg. (ENV) (Peris-Felipo et al. 2014a).

\section{Additional material examined}

DENMARK: 1 , , E Jutland, Albæk, NE of Hemmed, 5 Oct. 1997, Munk leg.; 1 , , E Jutland, Hessel near Grenå, 9 Aug. 1989, Munk leg.; 1 +, E Jutland, Højen Bæk, 5 km S of Vejle, 30 Aug. 1983, Munk leg.; 1 ㅇ, E Jutland, Højlund Skov, 5603' N, 9²4' E, 22 Aug. 1987, Munk leg.; 1 q, E Jutland, Nørreris Skov, 10 km NW of Aarhus, 28 Aug. 1985, Munk leg.; 1 ․ E Jutland, Yding Skov, 56 $00^{\prime}$ N, $9^{\circ} 48^{\prime}$ E, 19 Sep. 1985, Munk leg.; 2 우, E Jutland, Sillerup, 12 km SW of Silkeborg, 15 Sep. 1986 and 11 Sep. 2000, Munk leg.; 1 q, W Jutland, Nørholm Skov, 8 Jul. 1984, Munk leg.; 1 q, N Jutland, Rebild, 15 Sep. 1982, Munk leg. 


\section{Distribution}

Palaearctic Region (Peris-Felipo et al. 2014a).

\section{Published material}

Dinotrema oxybelum Munk \& Peris-Felipo, 2014

DENMARK: 1 + (paratype), W Jutland, Ammitsbøl Skov, 24 Jul. 1984, Munk leg. (NMA); 1 , E Jutland, Klattrup, 7 km S of Vejle, 19 Jul. 1983, Munk leg. (NMA); 1 क (paratype), E Jutland, Hørbylunde, 56ํํㅇ' N, 9²4' E, 22 Jun. 1987, Munk leg. (ENV) (Peris-Felipo et al. 2014a).

\section{Distribution}

Western Palaearctic (Peris-Felipo et al. 2014a). Only known from Denmark.

\section{Published material}

Dinotrema paludellae Munk \& Peris-Felipo, 2013

DENMARK: 1 (holotype), 2 우 (paratypes), E Jutland, Sillerup, $12 \mathrm{~km} \mathrm{SW}$ of Silkeborg, 19 and 24 May 1986, Munk leg. (NMA); 1 o (paratype), W Jutland, Bredsgårde, 56 27' N, $9^{\circ} 16^{\prime}$ E, 30 May 1985, Munk leg. (NMA); 1 \&, E Jutland, Klattrup, 7 km S of Vejle, 27 Aug. 1982, Munk leg. (ENV); 1 , , N Jutland, Villestrup, 56²46' N, 956' E, 15 Jun. 1984, Munk leg. (ENV) (Peris-Felipo et al. 2014a).

\section{Distribution}

Western Palaearctic (Munk et al. 2013b; Peris-Felipo et al. 2014a). Only known from Denmark.

\section{Published material}

Dinotrema paramicum Munk \& Peris-Felipo, 2013

DENMARK: 1 (holotype), 3 + $q$ (paratypes), E Jutland, Mols, Strandkær 56 $6^{\circ} 14^{\prime}$ N, $10^{\circ} 25^{\prime}$ E, 30 Sep. 1982, Munk leg. (ENV, NMA) (Peris-Felipo et al. 2014a).

\section{Distribution}

Palaearctic Region (Munk et al. 2013a; Peris-Felipo et al. 2014a).

\section{Published material}

Dinotrema pratense van Achterberg, 1988

DENMARK: 1 q, E Jutland, Skramsø Plantage, 56 ${ }^{\circ} 17^{\prime}$ N, 1040’ E, 4 Sep. 1989, Munk leg. (ENV) (Peris-Felipo et al. 2014a).

\section{Additional material examined}

DENMARK: 1 ð̊, N Jutland, Åstrup, 16 May 1982, Munk leg.; 1 ค, E Jutland, Klattrup, 4 Aug. 1984, Munk leg.; 1 ð̊, N Jutland, NJ 67, Slotved Skov, 20 Sep. 1982, swept on Lepiota sp., Munk leg.; 1 q, E Jutland, Skramsø Plantage, 56 $17^{\prime}$ N, 1040' E, 4 Sep. 1989, Munk leg.

\section{Distribution}

Palaearctic Region (Peris-Felipo et al. 2014a).

\section{Remarks}

One specimen was collected from Lepiota sp. (Fungi). 


\section{Published material}

Dinotrema propodeale (Tobias, 1962)

DENMARK: 4 qq, N Jutland, NJ77, Sindal, 25 Jul. 1985, Munk leg. (NHMW); 2 q $q$, E Jutland, Grejsdal, 1 km NE of Lerbæk Mølle, 9 Aug. 1985, Munk leg. (NHMW) (Peris-Felipo et al. 2014a).

\section{Distribution}

Palaearctic Region (Peris-Felipo et al. 2014a).

\section{Material examined}

*Dinotrema pulvinatum (Stelfox \& Graham, 1949)

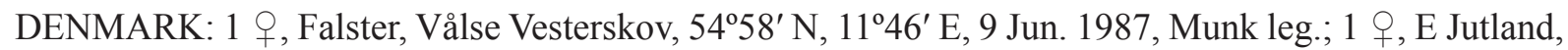
Hjelm, 3-5 Aug. 1992, Munk leg.; 1 q, E Jutland, Fuglslev, 56 $16^{\prime}$ N, 1043' E, 9 Aug. 1998, Munk

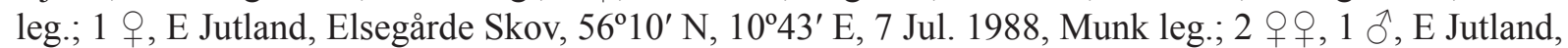
Glatved, 56 $17^{\prime}$ N, 10 ${ }^{\circ} 50^{\prime}$ E, 15 Aug. 1992 and 18 Aug. 1997, Munk leg.; 1 , E Jutland, Lykkesholm,

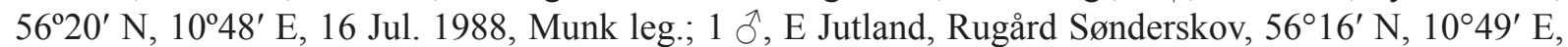
20 Jul. 1996, Munk leg.; 1 ô, E Jutland, Trelde Skov, N of Fredericia, 11 Jul. 1977, swept in old beech wood, Munk leg.; 1 ․, N Jutland, 3 km SW of Gerum, 21 Aug. 1997, swept on moor/edge of pond in Guldbækskær, Munk leg.; 1 đ’, N Jutland, Sæbygård Skov, 57²0' N, 10²9' E, 29 Aug. 1996, Munk leg.; 1 , , N Jutland, Vrangbæk, 5 km SW of Frederikshavn, 30 Aug. 1996, swept on wood-edge, Munk leg.

\section{Distribution}

Palaearctic Region (Yu et al. 2012). Denmark (first record).

\section{Remarks}

Some specimens were sampled from old beech wood and moor edge.

\section{Published material}

Dinotrema semicompressum (Stelfox \& Graham, 1949)

DENMARK: 1 ㅇ, E Jutland, Rugård Sønderskov, 56¹6' N, 1049’ E, 15 Sep. 1996, Munk leg. (ENV) (Peris-Felipo et al. 2014a).

\section{Distribution}

Palaearctic Region (Peris-Felipo et al. 2014a).

\section{Published material}

\section{Dinotrema significarium (Fischer, 1973)}

DENMARK: 6 우, E Jutland, Højen Bæk, 5 km S of Vejle, 1 and 4 Jun. 1984, Munk leg. (NHMW) (Peris-Felipo et al. 2014a).

\section{Material examined}

DENMARK: 1 + , E Jutland, Egå, 10 km NE Aarhus, 13-19 Sep. 1984, Munk leg.; 1 \&, E Jutland, Grejsdalen, Vejle, 1 km NE Lerbæk, 9 Aug. 1985, Munk leg.

\section{Distribution}

Palaearctic Region (Peris-Felipo et al. 2014a). 
Published material

Dinotrema tergitale (Fischer, 1973)

DENMARK: 2 q $q$, E Jutland, Hestenhave, S of Rønde, 20 Jul. 2000, Munk leg. (ENV) (Peris-Felipo et al. 2014a).

\section{Distribution}

Western Palaearctic (Peris-Felipo et al. 2014a).

\section{Published material}

Dinotrema thurnense (Fischer, 1977)

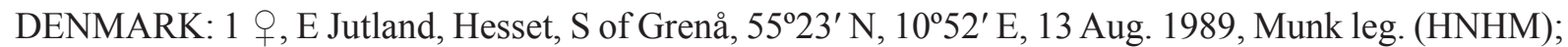
2 우, E Jutland, Højen Bæk, 5 km S of Vejle, 14 Jul. 1984 and 16 Jun. 1986, Munk leg. (NHMW); 1 ,

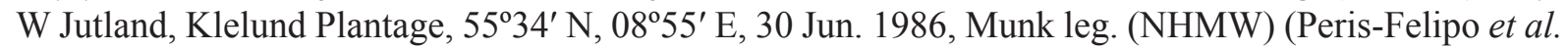
2014a).

\section{Material examined}

DENMARK: 1 q, E Jutland, Højen Bæk, 5 km S of Vejle, 15 Jun. 1989, Munk leg.

\section{Distribution}

Western Palaearctic (Peris-Felipo et al. 2014a).

\section{Published material}

Dinotrema valvulatum Munk \& Peris-Felipo, 2013

DENMARK: 1 (holotype), 1 (paratype), E Jutland, Højen Bæk, 5 km S of Vejle, 7 Jul. 1984, Munk leg. (NMA) (Peris-Felipo et al. 2014a).

\section{Distribution}

Palaearctic Region (Peris-Felipo et al. 2014a).

\section{Remarks}

Both specimens were collected in a moist wood with Alnus, Fraxinus and Fagus.

Genus Eudinostigma Tobias, 1986

\section{Material examined}

*Eudinostigma latistigma (Fischer, 1962)

DENMARK: 9 우, 3 $\hat{\jmath}$, E Jutland, Horsted, 7 km of Vejle, 31 May-2 Jun., 26 Jun., 5-9 Jul., and 2-4

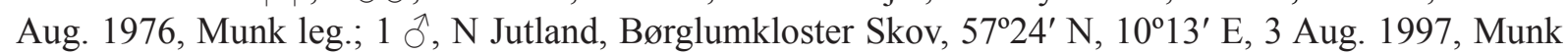
leg.

\section{Distribution}

Palaearctic Region (Yu et al. 2012). Denmark (first record).

Genus Orthostigma Ratzeburg, 1844

Published material

Orthostigma cratospilum (Thomson, 1895)

"Kb. [Dyrehaven, Zealand]" (Königsmann 1969). 


\section{Distribution}

Palaearctic Region (Yu et al. 2012).

\section{Published material}

Orthostigma laticeps (Thomson, 1895)

“Aasø, Langeland; Rudehegn, Zealand” (Königsmann 1969).

\section{Distribution}

Palaearctic Region (Yu et al. 2012).

\section{Published material}

Orthostigma longicubitale Königsmann, 1969

Data obtained from Fauna Europea (van Achterberg 2013).

\section{Distribution}

Oriental and Palaearctic Regions (Yu et al. 2012).

\section{Published material}

Orthostigma pumilum (Nees, 1834)

"Dänemark; Geelsskov, Zealand; Furesøen, Zealand; Dyrehaven, Zealand; Aasø, Langeland;" (Königsmann 1969).

\section{Distribution}

Palaearctic Region (Yu et al. 2012).

\section{Published material}

Orthostigma sordipes (Thomson, 1895)

“Als af Oberlehrer Wüstnei”, "NS [Geelsskov, Zealand]” (Thomson 1895; Marshall 1900; Königsmann 1969).

\section{Distribution}

Palaearctic Region (Yu et al. 2012).

Genus Pentapleura Forster, 1863

Published material

Pentapleura angustula (Haliday, 1838)

Data obtained from Fauna Europea (van Achterberg 2013).

\section{Distribution}

Palaearctic Region (Yu et al. 2012).

\section{Published material}

Pentapleura pumilo (Nees, 1812)

Data obtained from Fauna Europea (van Achterberg 2013).

\section{Distribution}

Nearctic, Oceanic and Palaearctic Regions (Yu et al. 2012). 
Genus Phaenocarpa Forster, 1863

Published material

Phaenocarpa conspurcator (Haliday, 1838)

"Dänemark" (Fischer 1970).

\section{Distribution}

Palaearctic Region (Yu et al. 2012).

\section{Published material}

Phaenocarpa impugnata Papp, 1972

"Stenholt Indelukke, Suserup Skov, and Frederikslund Skov" (Papp 1972).

\section{Distribution}

Palaearctic Region (Yu et al. 2012).

\section{Published material}

Phaenocarpa ruficeps (Nees, 1812)

Data obtained from Fauna Europea (van Achterberg 2013).

\section{Distribution}

Afrotropical, Nearctic, Oriental and Palaearctic Regions (Yu et al. 2012).

Genus Symphanes Foerster, 1863

\section{Published material}

Symphanes (Neosymphanes) striolata (Thomson, 1895)

"Denmark" (Koponen 2000).

\section{Distribution}

Western Palaearctic (Yu et al. 2012).

Genus Synaldis Foerster, 1863

\section{Material examined}

*Synaldis acutidentata Fischer, 1970

DENMARK: 2 우, E Jutland, Højkol Skov, 5605’ N, 9³8' E, 7 Jul. 1986 and 21 Jul. 1988, Munk leg.; 1 +, E Jutland, Ramten Skov, 17 Aug. 1997, Munk leg.; 1 \&, S Jutland, Arild, 1 Jul. 1986, Munk leg.

\section{Distribution}

Palaearctic Region (Yu et al. 2012). Denmark (first record).

\section{Published material}

Synaldis agaricae Munk \& Peris-Felipo, 2014

DENMARK: 9 우 (paratypes), N Jutland, Slotved Skov, 20 Sep. 1982, Munk leg. (NHMW, NMA. ZISP); 1 + 1 ô, N Jutland, Bøgsted Plantage, 16 Sep. 1982, Munk leg. (NMA); 1 +, W Jutland, Høgild, 24 Aug. 1985, Munk leg. (NMA); 9 우, 1 Oૈ, E Jutland, Mols, Strandkær, 4 Oct. 1983 and 3

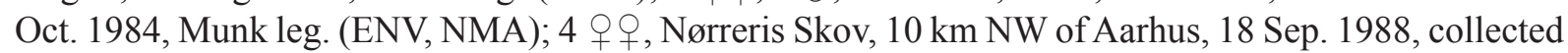


on Chlorophyllum rhacodes (V. Mahler) (NHMW, NMA); 4 우, N Jutland, Højen Bæk, 27 Sep. 1982, Munk leg. (NHMW, NMA) (Peris-Felipo et al. 2014b).

\section{Additional Material examined}

1 q, E Jutland, Klattrup, 27 Sep. 1982, Munk leg.

\section{Distribution}

Palaearctic Region (Peris-Felipo et al. 2014b).

\section{Remarks}

One specimen was collected from Agaricus nivescens (Fungi) and another one from Chlorophyllum rhacodes (Fungi).

\section{Material examined}

*Synaldis cultrigaster Fischer, 1970

DENMARK: 1 q, NE Zealand, Allindelille Skov, 14 Jul. 1986, Munk leg.; 2 q + , E Jutland, Nørreskov, $10 \mathrm{~km}$ E of Kolding, 18 Sep. 1982 and 5 Jul. 1987, Munk leg.

\section{Distribution}

Palaearctic Region (Yu et al. 2012). Denmark (first record).

\section{Published material}

Synaldis machiarum Munk \& Peris-Felipo, 2014

DENMARK: 1 (holotype), 5 qq (paratypes), E Jutland, Højen Bæk $5 \mathrm{~km} \mathrm{~S}$ of Vejle, 17 Jul. 1983, 5-6, 21, 30 Jul. 1984, and 18 Aug. 1984, Munk leg. (ENV, NMA. ZISP); 1 q (paratype), E Jutland, Vingsted, $10 \mathrm{~km} \mathrm{SW}$ of Vejle, 8 Jun. 1983, Munk leg. (NMA); 1 ( (paratype), E Jutland, Tirsbæk, $5 \mathrm{~km}$ E of Vejle, 18 Aug. 1984, Munk leg. (NMA); 1 (paratype), N Jutland, Børglumkloster Skov, 57² $24^{\prime}$ N, 10¹3' E, 1984, Munk leg. (NMA) (Peris-Felipo et al. 2014b).

\section{Distribution}

Palaearctic Region (Yu et al. 2012). Only known from Denmark.

\section{Material examined}

*Synaldis maxima Fischer, 1962

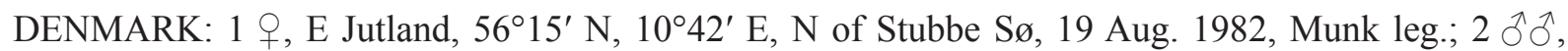
N Jutland, Nørlund, near Rold Skov, 25 km S of Alborg, 14 May 2000, Munk leg.; 2 우, E Jutland, Sillerup, 24 Jun. 1986 and 27 Jun. 1986, Munk leg.

\section{Distribution}

Palaearctic Region (Yu et al. 2012). Denmark (first record).

\section{Material examined}

*Synaldis megastigma Fischer, 1967

DENMARK: 1 q, E Jutland, Højkol Skov, 56º5’ N, 9³8' E, 15 Jul. 1987, Munk leg.

\section{Distribution}

Western Palaearctic (Yu et al. 2012). Denmark (first record). 
Genus Tanycarpa Forster, 1863

Published material

Tanycarpa gracilicornis (Nees, 1812)

Data obtained from Fauna Europea (van Achterberg 2013).

\section{Distribution}

Oriental and Palaearctic Regions (Yu et al. 2012).

Tribe Dacnusini Foerster, 1863

Genus Antrusa Nixon, 1943

Published material

Antrusa flavicoxa (Thomson, 1895)

"Randers (Jutland), Donse and Damhusmose (Zealand) and Copenhagen" (Griffiths 1967a).

\section{Distribution}

Palaearctic Region (Yu et al. 2012).

Genus Chaenusa Haliday, 1839

Published material

Chaenusa conjungens (Nees, 1811)

Data obtained from Fauna Europea (van Achterberg 2013).

\section{Distribution}

Palaearctic Region (Yu et al. 2012).

Genus Chorebus Haliday, 1833

Published material

Chorebus (Phaenolexis) abaris (Nixon, 1943)

"Utterslev Mose, Zealand" (Griffiths 1967b).

\section{Distribution}

Western Palaearctic (Yu et al. 2012).

\section{Published material}

Chorebus (Chorebus) affinis (Nees, 1811)

Data obtained from Fauna Europea (van Achterberg 2013).

\section{Distribution}

Palaearctic Region (Yu et al. 2012).

\section{Published material}

Chorebus (Stiphrocera) albipes (Haliday, 1839)

“Damhusmose, Utterslevmose, Køge (Zealand), Tranekær Hestehave, Langeland” (Griffiths 1967b). 


\section{Distribution}

Palaearctic Region (Yu et al. 2012).

\section{Published material}

Chorebus (Stiphrocera) aphantus (Marshall, 1896)

"Køge, Zealand; Damhusmose, Zealand; Aarhus, Jutland; Randers, Jutland" (Griffiths 1967b).

\section{Distribution}

Palaearctic Region (Yu et al. 2012).

\section{Published material}

Chorebus (Chorebus) bensoni (Nixon, 1943)

"Randers, Jutland; Damhusmose, Zealand" (Griffiths 1967b).

\section{Distribution}

Western Palaearctic (Yu et al. 2012).

\section{Published material}

Chorebus (Stiphocera) calthae Griffiths, 1967

"Randers, Jutland" (Griffiths 1967b).

\section{Distribution}

Western Palaearctic (Yu et al. 2012).

\section{Published material}

Chorebus (Stiphrocera) cinctus (Haliday, 1839)

Data obtained from Fauna Europea (van Achterberg 2013).

\section{Distribution}

Palaearctic Region (Yu et al. 2012).

\section{Published material}

Chorebus (Chorebus) crassipes (Stelfox, 1954)

"Randers, Jutland" (Griffiths 1967b).

\section{Distribution}

Palaearctic Region (Yu et al. 2012).

\section{Published material}

Chorebus (Stiphrocera) fallaciosae Griffiths, 1967

"Randers, Jutland" (Griffiths 1967b).

\section{Distribution}

Western Palaearctic (Yu et al. 2012). 


\section{Published material}

\section{Chorebus (Chorebus) flavipes (Goureau, 1851)}

“Copenhagen district, Zealand" (Griffiths 1968a).

\section{Distribution}

Western Palaearctic (Yu et al. 2012).

\section{Published material}

Chorebus (Chorebus) hilaris Griffiths, 1967

"Denmark" (Griffiths 1967a).

\section{Distribution}

Western Palaearctic (Yu et al. 2012).

Published material

Chorebus (Chorebus) humeralis Griffiths, 1968

"Rudehegn, Køge, Damhusmose, Zealand" (Griffiths 1968a).

\section{Distribution}

Western Palaearctic (Yu et al. 2012).

\section{Published material}

Chorebus (Chorebus) kama (Nixon, 1945)

"Køge, Rudehegn, Damhusmose and Utterslev Mose (Zealand); Randers (Jutland)" (Griffiths 1967b).

\section{Distribution}

Western Palaearctic (Yu et al. 2012).

\section{Published material}

Chorebus (Chorebus) lanigerus (Stelfox, 1957)

"Randers, Jutland “(Griffiths 1968a).

\section{Distribution}

Western Palaearctic (Yu et al. 2012).

\section{Published material}

Chorebus (Stiphrocera) misellus (Marshall, 1895)

Hoptrup (Kolding, Jutland) (Drea et al. 1982).

\section{Distribution}

Palaearctic Region (Yu et al. 2012).

\section{Published material}

Chorebus (Chorebus) nigriscaposus (Nixon, 1949)

"Randers and Aarhus, Jutland" (Griffiths 1968a). 


\section{Distribution}

Western Palaearctic (Yu et al. 2012).

\section{Published material}

Chorebus (Chorebus) nydia (Nixon, 1937)

“Damhusmose, Donse and Utterslev Mose (Zealand) and Copenhagen" (Griffiths 1967a).

\section{Distribution}

Palaearctic Region (Yu et al. 2012).

\section{Published material}

Chorebus (Chorebus) poemyzae Griffiths, 1968

"Donse, Zealand; Randers, Jutland; Damhusmose, Zealand; Utterslevmose, Zealand; Taastrup, Zealand; Koge, Zealand" (Griffiths 1968a).

\section{Distribution}

Western Palaearctic (Yu et al. 2012).

\section{Published material}

Chorebus (Chorebus) rufipes (Nees, 1812)

Data obtained from Fauna Europea (van Achterberg 2013).

\section{Distribution}

Palaearctic Region (Yu et al. 2012).

Published material

Chorebus (Stiphrocera) rotundiventris (Thomson, 1895)

"Donse, Zealand" (Griffiths 1967a).

\section{Distribution}

Western Palaearctic (Yu et al. 2012).

\section{Published material}

Chorebus (Stiphrocera) schlicki Griffiths, 1968

"Damhusmose, Zealand; Donse, Zealand; Utterslevmose, Zealand; Præstevangen, Frederiksborg, Zealand; Randers, Jutland" (Griffiths 1968b).

\section{Distribution}

Western Palaearctic (Yu et al. 2012). Only known from Denmark.

\section{Published material}

Chorebus (Chorebus) tamiris (Nixon, 1943)

"Randers, Jutland" (Griffiths 1967b).

\section{Distribution}

Western Palaearctic (Yu et al. 2012). 
Published material

Chorebus (Chorebus) tenellae Griffiths, 1967

"Randers, Jutland; Praestevangen, Zealand" (Griffiths 1967b).

\section{Distribution}

Western Palaearctic (Yu et al. 2012).

\section{Published material}

Chorebus (Chorebus) uliginosus (Haliday, 1839)

Data obtained from Fauna Europea (van Achterberg 2013).

\section{Distribution}

Palaearctic Region (Yu et al. 2012).

\section{Published material}

Chorebus (Stiphrocera) xanthaspidae Griffiths, 1968

"Rudehegn, Præstevangen, Frederiksborg, Zealand" (Griffiths 1968b).

\section{Distribution}

Palaearctic Region (Yu et al. 2012).

Genus Coelinius Nees, 1819

Published material

$$
\text { Coelinius parvulus (Nees, 1811) }
$$

“Dyrehaven (Copenhagen)” (Maréchal 1938).

\section{Distribution}

Palaearctic Region (Yu et al. 2012).

Genus Coloneura Forster, 1863

Published material

$$
\text { Coloneura ate (Nixon, 1943) }
$$

"Danish specimen" (Griffiths 1968b; Fischer 1997; Lozan et al. 2010).

\section{Distribution}

Western Palaearctic (Yu et al. 2012).

\section{Published material}

\section{Coloneura danica Griffiths, 1968}

“Utterslev Mose, Damhusmose, Zealand" (Griffiths 1968b; Fischer 1997; Lozan et al. 2010).

\section{Distribution}

Western Palaearctic (Yu et al. 2012). 


\title{
Published material
}

Coloneura major Griffiths, 1967

“Damhusmose, Zealand” (Griffiths 1967b; Fischer 1997).

\section{Distribution}

Western Palaearctic (Yu et al. 2012).

Genus Dacnusa Haliday, 1833

\section{Published material}

\author{
Dacnusa (Agonia) adducta (Haliday, 1839)
}

“Præstevangen, Frederiksborg, Zealand" (Griffiths 1968b).

\section{Distribution}

Palaearctic Region (Yu et al. 2012).

\section{Published material}

Dacnusa (Dacnusa) confinis Ruthe, 1859

"Køge and Utterslev Mose, Zealand" (Griffiths 1967b).

\section{Distribution}

Palaearctic Region (Yu et al. 2012).

\section{Published material}

Dacnusa (Pachysema) discolor (Foerster, 1862)

"Vester Fælled, Copenhagen" (Griffiths 1968b).

\section{Distribution}

Western Palaearctic (Yu et al. 2012).

\section{Published material}

Dacnusa (Dacnusa) dryas (Nixon, 1948)

"Hoptrup (Kolding, Jutland)" (Drea et al. 1982).

\section{Distribution}

Nearctic and Palaearctic Region (Yu et al. 2012).

\section{Published material}

Dacnusa (Pachysema) ergeteles (Nixon, 1954)

"Randers, Jutland" (Griffiths 1967b).

\section{Distribution}

Palaearctic Region (Yu et al. 2012).

\section{Published material}

Dacnusa (Pachysema) fasciata Stelfox, 1954

"Damhusmose, Zealand and Randers, Jutland" (Griffiths 1967b). 


\section{Distribution}

Western Palaearctic (Yu et al. 2012).

\section{Published material}

Dacnusa (Aphanta) hospita (Foerster, 1862)

"Randers, Jutland" (Griffiths 1967b).

\section{Distribution}

Palaearctic Region (Yu et al. 2012).

\section{Published material}

Dacnusa (Dacnusa) lugens (Haliday, 1839)

Data obtained from Fauna Europea (van Achterberg 2013).

\section{Distribution}

Palaearctic Region (Yu et al. 2012).

\section{Published material}

Dacnusa (Pachysema) macrospila (Haliday, 1839)

"Randers and Aalborg (Jutland), Køge, Damhusmose and Utterslev Mose (Zealand)" (Griffiths 1967b).

\section{Distribution}

Palaearctic Region (Yu et al. 2012).

Published material

Dacnusa (Dacnusa) maculipes Thomson, 1895

Hoptrup (Kolding, Jutland) (Drea et al. 1982).

\section{Distribution}

Palaearctic Region (Yu et al. 2012).

\section{Published material}

Dacnusa (Pachysema) monticola (Foerster, 1862)

“Præstevangen, Zealand; Randers, Jutland” (Griffiths 1967b).

\section{Distribution}

Palaearctic Region (Yu et al. 2012).

\section{Published material}

Dacnusa (Pachysema) nigrella Griffiths, 1967

“Randers, Jutland; Præstevangen, Zealand; Copenhagen" (Griffiths 1967b).

\section{Distribution}

Western Palaearctic (Yu et al. 2012). 


\section{Published material}

\section{Dacnusa (Pachysema) obesa Stelfox, 1954}

“Utterslev Mose, Darnhusmose, Køge and Donse, Zealand; Randers, Jutland” (Griffiths 1968b).

\section{Distribution}

Western Palaearctic (Yu et al. 2012).

\section{Published material}

Dacnusa (Pachysema) sibirica Telenga, 1935

"Vester Fælled, Copenhagen" (Griffiths 1967b).

\section{Distribution}

Palaearctic Region (Yu et al. 2012).

\section{Published material}

Dacnusa (Dacnusa) stramineipes (Haliday, 1839)

Data obtained from Fauna Europea (van Achterberg 2013).

\section{Distribution}

Palaearctic Region (Yu et al. 2012).

\section{Published material}

Dacnusa (Dacnusa) sulcata (Tobias, 1962)

"Randers, Jutland" (Griffiths 1967b).

\section{Distribution}

Palaearctic Region (Yu et al. 2012).

\section{Published material}

Dacnusa (Dacnusa) tarsalis Thomson, 1895

Data obtained from Fauna Europea (van Achterberg 2013).

\section{Distribution}

Western Palaearctic Region (Yu et al. 2012).

Genus Exotela Forster, 1863

Published material

$$
\text { Exotela gilvipes (Haliday, 1839) }
$$

"Randers, Jutland; Darnhusmose and Køge, Zealand" (Griffiths 1967b).

\section{Distribution}

Palaearctic Region (Yu et al. 2012). 
Genus Polemochartus Schulz, 1911

Published material

Polemochartus liparae (Giraud, 1863)

“Sønderborg af Oberlehrer Wüstnei” (Thomson 1895).

\section{Distribution}

Palaearctic Region (Yu et al. 2012).

Genus Protodacnusa Griffiths, 1964

Published material

Protodacnusa litoralis Griffiths, 1964

Data obtained from Fauna Europea (van Achterberg 2013).

\section{Distribution}

Palaearctic Region (Yu et al. 2012).

Genus Trachionus Haliday, 1833

Published material

Trachionus (Planiricus) hians (Nees, 1816)

"Denmark" (Zaykov 1982).

\section{Distribution}

Palaearctic Region (Yu et al. 2012).

\section{Published material}

Trachionus (Trachionus) mandibularis (Nees, 1816)

"Denmark" (Zaykov 1982).

\section{Distribution}

Palaearctic Region (Yu et al. 2012).

\section{Published material}

Trachionus (Planiricus) ringens (Haliday, 1839)

"Denmark" (Zaykov 1982).

\section{Distribution}

Palaearctic Region (Yu et al. 2012).

\section{Discussion}

Alysiinae is one of the largest subfamilies within Braconidae and numerous species remain to be described around the World (Peris-Felipo et al. 2014a). Recently, several Alysiinae revisions and catalogues have been published for different genera (Peris-Felipo et al. 2012, 2014a, c; 2015, 2016; Khajeh et al. 2014; Gadallah et al. 2015; Peris-Felipo 2015; Peris-Felipo \& Belokobylskij 2016a, b). However, few studies of Alysiinae from the Nordic countries have been carried out (Munk et al. 2013a, b): only 29 species are known from Iceland, 45 species from Finland and Norway, and 234 from Sweden (Yu et al. 2012). 
In the present study, a total of 153 alysiine species have been catalogued from Denmark (excluding the Faroe Islands and Greenland) with two species described as new for science, Adelphenaldis leptoarticulata sp. nov. and A. grandis sp. nov. However, this number of species is still far from the numbers of better studied adjacent countries: Germany (337), Poland (231), Sweden (234) or the Netherlands (203) (Yu et al. 2012; van Achterberg 2013).

Additionally, 38 species are recorded from Denmark for the first time: Adelphenaldis globipes (Fischer, 1962), Alysia (Anarcha) sophia Haliday, 1838, Aspilota delicata Fischer, 1973, A. furtnerana Fischer, 1973, A. fuscicornis (Haliday, 1838), A. hirticornis (Thomson, 1895), A. imparidens Fischer, 1974, A. insolita (Tobias, 1962), A. intermediana Fischer, 1975, A. iocosipecta Fischer, 1974, A. iuxtanaeviam Fischer, 1980, A. laevinota Tobias, 1962, A. nidicola Hedqvist, 1972, A. pillerensis Fischer, 1973, A. stenogaster Stelfox \& Graham, 1951, A. styriaca Fischer, 1973, A. tetragona Fischer, 1976, A. ventasa Belokobylskij, 2007, A. vernalis Stelfox \& Graham, 1951, Dinotrema concinnum (Haliday, 1838), D. contracticorne (Fischer, 1974), D. cratocera (Thomson, 1895), D. cruciatum (Fischer, 1973), D. cruciforme (Fischer, 1973), D. denticulatum (Stelfox \& Graham, 1951), D. (Leptotrema) dentifemur (Stelfox, 1943), D. divisum (Stelfox \& Graham, 1950), D. hodiense (Fischer, 1976), D. latifemur (Fischer, 1975), D. mesocaudatum van Achterberg, 1988, D. microcera (Thomson, 1895), D. nigricorne (Thomson, 1985), D. oleraceum (Tobias, 1962), D. pulvinatum (Stelfox \& Graham, 1949), Eudinostigma latistigma (Fischer, 1962), Synaldis acutidentata Fischer, 1970, S. cultrigaster Fischer, 1970, S. maxima Fischer, 1962 and S. megastigma Fischer, 1967.

In general, the limited number of known Alysiinae from Denmark reflects the paucity of research on this group. Further investigations both on the faunal composition and mainly host associations of Alysiinae are necessary to evaluate their role as biological control of dipterous pests in agricultural and urban landscapes.

\section{Acknowledgements}

The authors are very grateful to Thorkild Munk's family for donating Munk's material to the Natural History Museum of Denmark (Copenhagen, Denmark; NHMD). This work was partly supported for the second author by the Russian Foundation for Basic Research (projects Nos 15-29-02466 and 16-0400197) and the Russian State Research Project No. 01201351189.

\section{References}

Belokobylskij S.A. 2005. On the systematic and distribution of three rare alysiine genera (Hymenoptera: Braconidae: Alysiinae). Genus (Wroclaw) 16 (3): 431-444.

Belokobylskij S.A. \& Tobias V.I. 2007. Alysiinae [in Russian]. In: Lelej A.S. (ed.) Key to the Insects of the Russian Far East. Vol. Apr. Neuropteroidea, Mecoptera, Hymenoptera. Pt 5: 9-133. Dalnauka, Vladivostok.

Berry J.A. 2007. Alysiinae (Insecta: Hymenoptera: Braconidae). Fauna of New Zealand 58: 3-94.

Danish Metereological Institute 2016. Available from http://www.dmi.dk/en/vejr/ [accessed 10 Nov. 2016]

Den Store Danske 2016. Available from http://denstoredanske.dk/Danmarks_geografi_og_historie/ Danmarks_geografi/Danmark_generelt/Danmark [accessed 28 Feb. 2016].

Drea J.J., Jeandel D. \& Gruber F. 1982. Parasites of agromyzid leafminers (Diptera: Agromyzidae) on alfalfa in Europe. Annals of the Entomological Society of America 75 (3): 297-310.

Elpino-Campos A., Del-Claro K. \& Prezoto F. 2007. Diversity of social wasps (Hymenoptera: Vespidae) in Cerrado fragments of Uberlandia, Minas Gerais state, Brazil. Neotropical Entomology 36: 685-692. 
Fischer M. 1970. Zur Kenntnis der europäischen Phaenocarpa-Arten mit besonderer Berücksichtigung der Fauna Niederösterreichs (Hymenoptera, Braconidae, Alysiinae). Zeitschrift für Angewandte Zoologie 57: 409-498.

Fischer M. 1972. Erste Gliederung der paläarktischen Aspilota-Arten (Hymenoptera, Braconidae, Alysiinae). Polskie Pismo Entomologiczne 42: 323-459.

Fischer M. 1974a. Studien an Alysiinen-Typen (Hymenoptera, Braconidae, Alysiinae). Zeischrift der Arbeitsgemeinschaft Österreichischer Entomologen 25: 47-51.

Fischer M. 1974b. Einige neue Aspilota-Arten aus Niederösterreich (Hymenoptera: Braconidae: Alysiinae). Folia Entomologica Hungarica 27: 59-71.

Fischer M. 1997. Taxonomische Untersuchungen über Kieferwespen (Insecta: Hymenoptera: Braconidae: Alysiinae) der Alten Welt. Annalen des Naturhistorischen Museums in Wien, Serie B, Botanik und Zoologie 99B: 97-143.

Fischer M. \& Beyarslan A. 2012. New species of Synaldis Foerster and Idiasta Foerster, and further records of Turkish Alysiini (Hymenoptera: Braconidae: Alysiinae). Zoology in the Middle East 55: 5564. http://dx.doi.org/10.1080/09397140.2012.10648918

Gadallah N., Ghahari H., Peris-Felipo F.J. \& Fischer M. 2015. A. annotated catalogue of the Iranian Alysiinae (Hymenoptera: Braconidae). Zootaxa 3974 (1): 1-28. http://dx.doi.org/10.11646/ zootaxa.3974.1.1

Gannota E. 1993. Five species of Chorebus (Hymenoptera, Braconidae, Alysiinae, Dacnusini) new to Finland. Entomologica Fennica 4 (1): 13.

Geodatastyrelsen 2016. Danish Geodata Agency [online]. Available from http://gst.dk/ [accessed 29 Jan. 2016].

Gimeno C., Belshaw R. \& Quicke D.L.J. 1997. Phylogenetic relationships of the Alysiinae/Opiinae (Hymenoptera: Braconidae) and the utility of cytochorome b, 16S and 28S D2 rRNA. Insect Molecular Biology 6 (3): 273-284. http://dx.doi.org/10.1046/j.1365-2583.1997.00181.x

González J.A., Tomé M.A.M., Gayubo S.F. \& Torres F. 2000. Himenópteros aculeados capturados mediante trampas Malaise en un sector arenoso de la Submeseta Norte (España) (Hymenoptera, Aculeata). Nouvelle Revue d'Entomologie 17: 337-353.

Griffiths G.C.D. 1967a. The Alysiinae (Hym. Braconidae) parasites of the Agromyzidae (Diptera) II. The parasites of Agromyza Fallén. Beiträge zur Entomologie 16 (5/6): 551-605.

Griffiths G.C.D. 1967b. The Alysiinae (Hym. Braconidae) parasites of the Agromyzidae (Diptera) III. The parasites of Paraphythomyza Enderlein, Phytagromyza Hendel and Phythomyza Fallén. Beiträge zur Entomologie 16 (7/8): 775-951.

Griffiths G.C.D. 1968a. The Alysiinae (Hym. Braconidae) parasites of the Agromyzidae (Diptera) VI. The parasites of Cerodontha Rondani s.1. Beiträge zur Entomologie 18 (1/2): 63-152.

Griffiths G.C.D. 1968b. The Alysiinae (Hym. Braconidae) parasites of the Agromyzidae (Diptera) V. The parasites of Liriomyza Mik and certain small genera of Phytomyzinae. Beiträge zur Entomologie $18(1 / 2): 5-62$.

Hedqvist K.J. 1972. Two new species of Aspilota Först. (Hym., Ichneumonoidea, Braconidae). Entomologisk Tidskrift 93: 216-219.

Hedqvist K.J. 1973. Two new species of Alysiinae Först. from north Sweden (Hym., Ichneumonoidea, Braconidae). Entomologisk Tidskrift 94: 91-93. 
Khajeh N., Yari Z., Rakhshani E. \& Peris-Felipo F.J. 2014. A regional checklist of Alysiinae (Hymenoptera: Braconidae) from Iran. Journal of Crop Protection 3 (3): 413-423.

Königsmann E. 1969. Beitrag zur Revision der Gattung Orthostigma (Hymenoptera, Braconidae). Deutsche Entomologische Zeitschrift 16 (I/III): 1-53.

Koponen M. 1999. Two species of Trachyusa Ruthe, 1854 (Hymenoptera, Braconidae, Alysiinae) in Finland. Entomologica Fennica 10: 199-200.

Koponen M. 2000. Five Alysiinae species new to Finland (Hymenpotera: Braconidae: Alysiinae). Entomologica Fennica 11: 239-243.

Koponen M. \& Vikberg V. 2013. Suomen Dacnusini-lajien luettelo [Check list of the species of Dacnusini in Finland] (Hymenoptera, Braconidae, Alysiinae). Sahlbergia 20: 2-27.

Lozan A., Belokobylskij S., van Achterberg C. \& Monaghan M.T. 2010. Diversity and distribution of Braconidae, a family of parasitoid wasps in the Central European peatbogs of South Bohemia, Czech Republic. Journal of Insect Science 10 (16): 1-21.

Maréchal P. 1938. Sur trois Coelinius de la collection Thomson (Hymén., Braconidae, Dacnusinae). Bulletin et Annales de la Société entomologique de Belgique 78 : 201-229.

Marshall T.A. 1900. Les Braconides (Supplément). In: André E. (ed.) Espèces des Hymenoptères d'Europe et d'Algerie. Tome 5 bis, V. Dubosclard, Paris.

Munk T., Peris-Felipo F.J. \& Jiménez-Peydró R. 2013a. New western Palaearctic Dinotrema species with mesoscutal pit and only medially sculptured propodeum (Hymenoptera, Braconidae, Alysiinae). Zookeys 260: 61-76. http://dx.doi.org/10.3897/zookeys.260.4084

Munk T., Peris-Felipo F.J. \& Jiménez-Peydró R. 2013b. New western Palaearctic species of the genus Dinotrema Foerster, 1862 with widely sculptured propodeum (Hymenoptera: Braconidae: Alysiinae). Annales Zoologici 63 (1): 123-141. http://dx.doi.org/10.3161/000345413x666174

Nielsen L.B. 1994. Distribution and population densities of stemboring fly larvae (Diptera) and their parasitoids in Danish farmland. Journal of Applied Entomology 117 (4): 321-331.

Papp J. 2008. Seven new species of Aspilota Foerster from the Palaearctic Region (Hymenoptera: Braconidae: Alysiinae). Annales Historico-Naturales Musei Nationalis Hungarici 100: 245-269.

Peris-Felipo F.J. 2015. Review of the genus Aphaereta Förster, 1863 (Hymenoptera, Braconidae, Alysiinae) from the Afrotropical region, with description of three new species. Journal of Hymenoptera Research 42: 1-20. http://dx.doi.org/10.3897/JHR.42.9135

Peris-Felipo F.J. \& Belokobylskij S.A. 2016a. First record of the genus Dinotrema Foerster, 1862 (Hymenoptera, Braconidae, Alysiinae) from the Neotropical region, with description of four new species and a key to the New World taxa. European Journal of Taxonomy 179: 1-23. http://dx.doi.org/10.5852/ ejt.2016.179

Peris-Felipo F.J. \& Belokobylskij S.A. 2016b. Afrotropical species of the genus Dinotrema Foerster, 1862 (Hymenoptera, Braconidae, Alysiinae) with the description of three new taxa and a key for determination. Bulletin of Insectology 69 (1): 93-106.

Peris-Felipo F.J., Belokobylskij S.A. \& Jiménez-Peydró R. 2012. Genus Adelphenaldis Fischer, 2003 (Hymenoptera: Braconidae: Alysiinae) in Spain, with a key to the World species. Annales Zoologici 62 (2): $287-296$.

Peris-Felipo F.J., Belokobylskij S.A. \& Jiménez-Peydró R. 2014a. Revision of the western Palaearctic species of the genus Dinotrema Foerster, 1862 (Hymenoptera, Braconidae, Alysiinae). Zootaxa 3885 (1): 1-483. http://dx.doi.org/10.11646/zootaxa.3885.1.1 
Peris-Felipo F.J., Belokobylskij S. \& Munk T. 2014b. Three new species of the genera Aspilota Foerster and Synaldis Foerster from North Europe (Hymenoptera: Braconidae: Alysiinae). Annales Zoologici 64 (4): 683-692.

Peris-Felipo F.J., Belokobylskij S.A., Falcó-Garí J. \& Jiménez-Peydró R. 2014c. Aspilota-group (Hymenoptera: Braconidae: Alysiinae) diversity in Mediterranean Natural Parks of Spain. Biodiversity Data Journal 2: e1112. http://dx.doi.org/10.3897/BDJ.2.e1112

Peris-Felipo F.J., Ameri A., Talebi A.A. \& Belokobylskij S.A. 2015. Review of the genus Aristelix Nixon, 1943 (Hymenoptera, Braconidae, Alysiinae), with description of a new species from Iran and clarification of the status of Antrusa chrysogastra (Tobias, 1986). Journal of Hymenoptera Research 45: 97-111. http://dx.doi.org/10.3897/JHR.45.5313

Peris-Felipo F.J., Yari Z., van Achterberg C., Rakhshani E. \& Belokobylskij S.A. 2016. Review of species of the genus Adelurola Strand, 1928, with a key to the species (Hymenoptera, Braconidae, Alysiinae). Zookeys 566: 13-30. http://dx.doi.org/10.3897/zookeys.566.6684

Riedel M., Hansen L.O. \& Berg Ø. 2002. Braconidae (Hymenoptera) of Norway, Part 1. Norwegian Journal of Entomology 49: 97-108.

Sharkey M.J. \& Wharton R.A. 1997. Morphology and terminology. In: Wharton R.A., Marsh P.M. \& Sharkey M.J. (eds) Manual of the New World Genera of the Family Braconidae (Hymenoptera): 19-37. International Society of Hymenopterists, Washington DC.

Shenefelt R.D. 1974. Braconidae 7: Alysiinae-Hymenopterorum Catalogus (new edition), Part II: 9371113. W. Junk, The Hague.

Thomson C.G. 1895. LII. Bidrag till Braconidernas Kännedom. Opuscula Entomologica 20: 2141-2339.

Tobias V.I. 1962. The material to alysiine fauna (Hymenoptera, Braconidae, Alysiinae) of the Leningrad Province. Proceedings of Zoological Institute of the USSR Academy of Sciences 31: 81-137.

Tobias V.I. 1986a. Order Hymenoptera, family Braconidae (2). Key to the insects of the European part of the USSR, III/5: 1-309.

Tobias V.I. 1986b. Keys to the insects of the European part of the USSR, Vol. III. Hymenoptera. Part V. Nauka, Leningrad.

van Achterberg C. 1993. Illustrated key to the subfamilies of the Braconidae (Hymenoptera: Ichneumonoidea). Zoologische Verhandelingen 283: 1-189.

van Achterberg C. 2013. Fauna Europaea: Alysiinae. In: Karsholt O. \& Nieukerken E.J. van. 2013. Fauna Europaea: Hymenoptera, Braconidae. Fauna Europaea version 2.6.2. Available from http://www. faunaeur.org [accessed 10 Nov. 2016].

van Achterberg C. \& Koponen M. 2003. Phaenocarpa ungulosetosa spec. nov. from Finland and Elasmosoma depressum spec. nov. from Estonia (Hymenoptera: Braconidae: Alysiinae: Alysiini, Euphorinae: Neoneurini). Zoologische Mededelingen 77 (16): 291-299.

van Achterberg C. \& Vikberg V. 2014. Dinotrema brevis spec. nov. (Hymenoptera: Braconidae: Alysiinae), a new brachypterous species from Finland. Zoologische Mededelingen 88 (1): 1-7.

Wharton R.A. 1986. The braconid genus Alysia (Hymenoptera): a description of the subgenera and a revision of the subgenus Alysia. Systematic Entomology 11: 453-504.

Wharton R.A. 1988. The braconid genus Alysia (Hymenoptera): a revision of the subgenus Anarcha. Contributions of the American Entomology Institute 25 (2): 1-69. 
Wharton R.A., Marsh P.M. \& Sharkey M.J. 1997. Manual of the New World Genera of the Family Braconidae (Hymenoptera). Special Publication 1, International Society of Hymenopterists, Washington DC.

Yu D.S., van Achterberg C. \& Horstman K. 2012. Taxapad 2012, Ichneumonoidea 2011. Database on flash-drive. Ottawa, Ontario, Canada.

Zaldivar-Riverón A., Mori M. \& Quicke D.L.J. 2006. Systematics of the cyclostome subfamilies of braconid parasitic wasps (Hymenoptera: Ichneumonoidea): a simultaneous molecular and morphological Bayesian approach. Molecular Phylogenetics and Evolution 38 (1): 130-145. http://dx.doi.org/10.1016/j. ympev.2005.08.006

Zaykov A.N. 1982. The European species of Symphyta Förster (Hymenoptera: Braconidae). Acta Zoologica Hungarica 28: 171-179.

Manuscript received: 19 April 2016

Manuscript accepted: 8 June 2016

Published on: 12 December 2016

Topic editor: Gavin Broad

Desk editors: Jeroen Venderickx and Laurence Bénichou

Printed versions of all papers are also deposited in the libraries of the institutes that are members of the EJT consortium: Muséum national d'Histoire naturelle, Paris, France; Botanic Garden Meise, Belgium; Royal Museum for Central Africa, Tervuren, Belgium; Natural History Museum, London, United Kingdom; Royal Belgian Institute of Natural Sciences, Brussels, Belgium; Natural History Museum of Denmark, Copenhagen, Denmark; Naturalis Biodiversity Center, Leiden, the Netherlands. 